\title{
SIMBA survey of southern high-mass star forming regions
}

\section{Physical parameters of the 1.2-mm/IRAS sources ${ }^{\star}$}

\author{
S. Faúndez ${ }^{1}$, L. Bronfman ${ }^{1}$, G. Garay ${ }^{1}$, R. Chini ${ }^{2}$, L.-Å. Nyman $^{3}$, and J. May ${ }^{1}$ \\ 1 Departamento de Astronomía, Universidad de Chile, Casilla 36-D, Santiago, Chile \\ e-mail: [leo; sfaundez]@das.uchile.cl \\ 2 Astronomisches Institut der Ruhr-Universität Bochum, Universitätsstr. 150/NA 7, 44780 Bochum, Germany \\ ${ }^{3}$ European Southern Observatory, Casilla 19001, Santiago, Chile
}

Received 27 November 2004 / Accepted 1 June 2004

\begin{abstract}
We report the results of a $1.2 \mathrm{~mm}$ continuum emission survey toward 146 IRAS sources thought to harbour highmass star forming regions. The sources have FIR colors typical of UCHII regions and were detected in the CS $(2 \rightarrow 1)$ line survey of Bronfman et al. (1996). Regions of $15^{\prime} \times 10^{\prime}$, centered on each IRAS source, were mapped with an angular resolution of $\sim 24^{\prime \prime}$, using the SIMBA array on the SEST telescope. $1.2 \mathrm{~mm}$ emission was detected toward all IRAS sources. We find that the dust cores associated with these sources have typical sizes of $0.4 \mathrm{pc}$ and masses of $5 \times 10^{3} \mathrm{M}_{\odot}$. Dust temperatures and luminosities, derived from the SED, are typically $32 \mathrm{~K}$ and $2.3 \times 10^{5} L_{\odot}$.
\end{abstract}

Key words. ISM: clouds - stars: formation - ISM: dust, extinction - radio continum: ISM - stars: circumstellar matter ISM: H II regions

\section{Introduction}

The process of formation of massive stars is still not well understood (e.g. Garay \& Lizano 1999). There are two main hypotheses to explain their mass build-up: (i) through coalescense of low-mass stars in high stellar density clusters $(n \geq$ $10^{8}$ stars $^{-3}$; Bonnell et al. 1998) and (ii) through accretion (Osorio \& Lizano 1999; Yorke \& Sonnhalter 2002; McKee \& Tan 2003). Recent observations of disks (Shepherd et al. 2001) and jets (Garay et al. 2003 ) give support to the accretion scenario, but coalescence cannot be ruled out.

Understanding the formation and early evolution of massive stars requires knowledge of the physical conditions of their birth environment. Due to the intrinsic scarcity of highmass stars and the short timescales involved in their formation $\left(t \sim 10^{5} \mathrm{yr}\right.$, McKee \& Tan 2003), the early stages of such formation have been only recently explored by systematic surveys (Plume et al. 1992, 1997; Bronfman et al. 1996; Beuther et al. 2002; Sridharan et al. 2002; Mueller et al. 2002). From a survey of molecular emission, in several transitions of CS, toward regions of massive star formation associated with water masers, Plume et al. (1997) found that high-mass stars are formed in molecular cores with typical densities of $6 \times 10^{5} \mathrm{~cm}^{-3}$, radii of $0.5 \mathrm{pc}$ and virial masses of $4 \times 10^{3} M_{\odot}$.

$\star$ Table 1 and Figs. 6 to 23 are only available in electronic form at http://www. edpsciences.org
With the goal of determining the physical characteristics of molecular cores harboring recently formed massive stars, at spatial scales of $\sim 0.5 \mathrm{pc}$, we have made a survey of $1.2 \mathrm{~mm}$ continuum emission toward 146 objects, thought to harbour high-mass star forming regions, using the SIMBA bolometer at SEST (Nyman et al. 2001). The sources were selected from the $\mathrm{CS}(2 \rightarrow 1)$ survey of Bronfman et al. (1996) ensuring high density gas and that they have FIR colours typical of ultra compact H II regions (Wood \& Churchwell 1989). The 1.2-mm emission was mapped within regions of $\sim 15^{\prime} \times 10^{\prime}$ centered on the IRAS sources. Moreover, since the sites of massive star formation are known to be gregarious, a further goal of this survey was to find massive cores in early stages of evolution, previous to the formation of a central massive object. In this paper we present the basic data set of the 1.2-mm objects associated with the IRAS sources. The identification of dense, cold and massive pre-stellar objects from this survey will be the subject of a further publication.

\section{Observations}

The observations were made using the 37-channel hexagonal bolometer array SIMBA installed at the SEST $15 \mathrm{~m}$ telescope. The passband of the bolometer has an equivalent width of $90 \mathrm{GHz}$ and is centered at $250 \mathrm{GHz}$. The SEST telescope has a beamwidth of $24^{\prime \prime}$ at the frequency band of SIMBA. The $1.2 \mathrm{~mm}$ emission was mapped toward 146 regions, 
centered on IRAS sources. We observed typically 2-3 maps per source, with a final rms of $40 \mathrm{mJy}^{\text {beam }}{ }^{-1}$. Typical map sizes were $900^{\prime \prime} \times 600^{\prime \prime}$. The maps were observed in the fast mapping mode in three runs between June 2001 and July 2002. The scans are in azimuth at a velocity of $80^{\prime \prime} / \mathrm{s}$, separated in elevation by $8^{\prime \prime}$. Data reduction was done using the MOPSI (Zylka 1998) software in three steps. In the first step, a standard procedure was followed to create an intensity map and to define a proper baseline to avoid artifacts close to the source. In the second stage, a model of the source is created using the previous map, which is used in the correlated sky noise filtering of the last step. Care was taken in choosing the intensity of the despiking because some sources were too strong. Sky opacity corrections were applied and the maps were calibrated from observations of Uranus or Mars. The calibration uncertainties are estimated at $20 \%$.

\subsection{Source selection}

The 146 observed IRAS sources were chosen as follows. We selected the brightest CS sources $\left(T_{A}>2.0 \mathrm{~K}\right)$ in the Bronfman et al. (1996) survey. Of the near 200 sources fulfilling this criteria, we observed 103 that homogeneously cover the galactic longitude. We complemented this sample with 34 sources that exhibit wings in the CS spectrum, indicative of outflow phenomena, and 9 sources without detections in the radio continuum survey of Walsh et. al (1998). The total number of sources brighter than $2.0 \mathrm{~K}$ is 116 , exhibiting broad wings in the $\mathrm{CS}(2 \rightarrow 1)$ line is 82 and with no radio continuum detections is 21 . The Galactic coverage of our survey is shown in Fig. 1. The majority of the sources are within the solar circle $(95 \%)$.

\subsection{Distances}

Kinematical distances were derived using the $\operatorname{CS}(2 \rightarrow 1)$ line velocities reported by Bronfman et al. (1996), a flat rotation curve, and the standard IAU constants for the solar velocity and solar radius of, respectively, $220 \mathrm{~km} \mathrm{~s}^{-1}$ and $8.5 \mathrm{kpc}$. The twofold ambiguity in kinematic distance that affects sources within the solar circle was resolved using a standard spiral arms model following Bronfman et al. (2000). For sources in the I quadrant, we adopted distances from the literature and, when not avilable, adopted the near distance. The nearest source is located at $400 \mathrm{pc}$ and the farthest one is at $13 \mathrm{kpc}$. The average distance is $4.4 \mathrm{kpc}$. The distribution of distances is shown in the lower right panel of Fig. 3

\section{Results}

1.2-mm emission was detected toward all 146 observed regions (each $\sim 15^{\prime} \times 10^{\prime}$ in size). Maps are shown in Figs. 6-22. In almost half of the maps we detected two or more objects (average of 2.2 sources per map. Figure 5 shows the distribution of number of sources per map). In particular we find that all IRAS sources are associated with $1.2 \mathrm{~mm}$ emission. The observed and derived parameters of the $1.2-\mathrm{mm}$ sources associated with the IRAS sources are summarized in Table 1. Column 1 gives the
IRAS name; Col. 2 the distance; Col. 3 the number of sources per map; Cols. 4 and 5 the right ascension and declination of the peak in the 1.2-mm emission; Col. 6 the 1.2-mm flux density; Col. 7 the angular size of the $1.2-\mathrm{mm}$ emission. The derived parameters, dust temperature, mass, density and luminosity, are given in Cols. 8 to 11, respectively. In what follows we describe how the value of the parameters were obtained.

\subsection{Sizes}

The angular sizes, given in Col. 7 of Table 1, correspond to the geometrical mean of the observed semi-major and semi-minor axes of Gaussians fitted to the 1.2-mm emission. An histogram of the distribution of the physical sizes, determined from the angular sizes using the distances listed in Col. 2, is shown in the upper left panel of Fig. 3. The average radius of the sources in our survey is $0.4 \pm 0.2 \mathrm{pc}$.

\subsection{Temperatures}

Dust temperatures were derived by fitting the spectral energy distribution (SED) using a modified blackbody model,

$S_{v}=\Omega B_{v}\left(T_{\mathrm{d}}\right)\left\{1-\exp \left(-\tau_{v}\right)\right\}$

where $S_{v}, B_{v}, \Omega$ and $T_{\mathrm{d}}$ are respectively the flux density, the Planck function, the angular size of the source and the dust temperature. We assume that the dependence of the optical depth, $\tau_{v}$, with frequency, $v$, can be expressed as

$\tau_{v}=\left(\frac{v}{v_{0}}\right)^{\beta}$,

where $\beta$ is the power law index and $v_{0}$ is the frequency at which the optical depth is unity, hereafter refereed as the turnover frequency.

A model with two dust components at different temperatures had to be used to obtain a good fit of the SED (consisting of the $1.2 \mathrm{~mm}$ flux and the fluxes in the four IRAS bands; see Fig. 2). We adopted as angular size of the colder component that given in Table 1 . We find that the cold component has a typical temperature of $\sim 32 \mathrm{~K}$, a turnover frequency of $\sim 3 \times 10^{3} \mathrm{GHz}$, and a power law index between $1.5-2.5$. The warmer component has a typical temperature of $\sim 140 \mathrm{~K}$ and a turnover frequency of $\sim 2 \times 10^{4} \mathrm{GHz}$ ( $\beta$ was fixed to 1 ). The angular size of this component was estimated from the fit. An histogram of the temperatures for the cold component is shown in the upper right panel of Fig. 3.

\subsection{Masses and densities}

The dust clouds in our survey have $N_{\mathrm{H}}\left(=N(\mathrm{H})+2 N\left(\mathrm{H}_{2}\right)\right)$ column densities considerably smaller than $10^{26} \mathrm{~cm}^{-2}$, the value at which $\tau_{1.2 \mathrm{~mm}} \sim 1$ for a temperature of $\approx 32 \mathrm{~K}$ (e.g. Mezger 1994). Therefore, the emission is optically thin and the dust mass can be computed using the expression (e.g. Chini et al. 1987),

$M_{\mathrm{d}}=\frac{S_{v} D^{2}}{k_{v} B_{v}\left(T_{\mathrm{d}}\right)}$, 

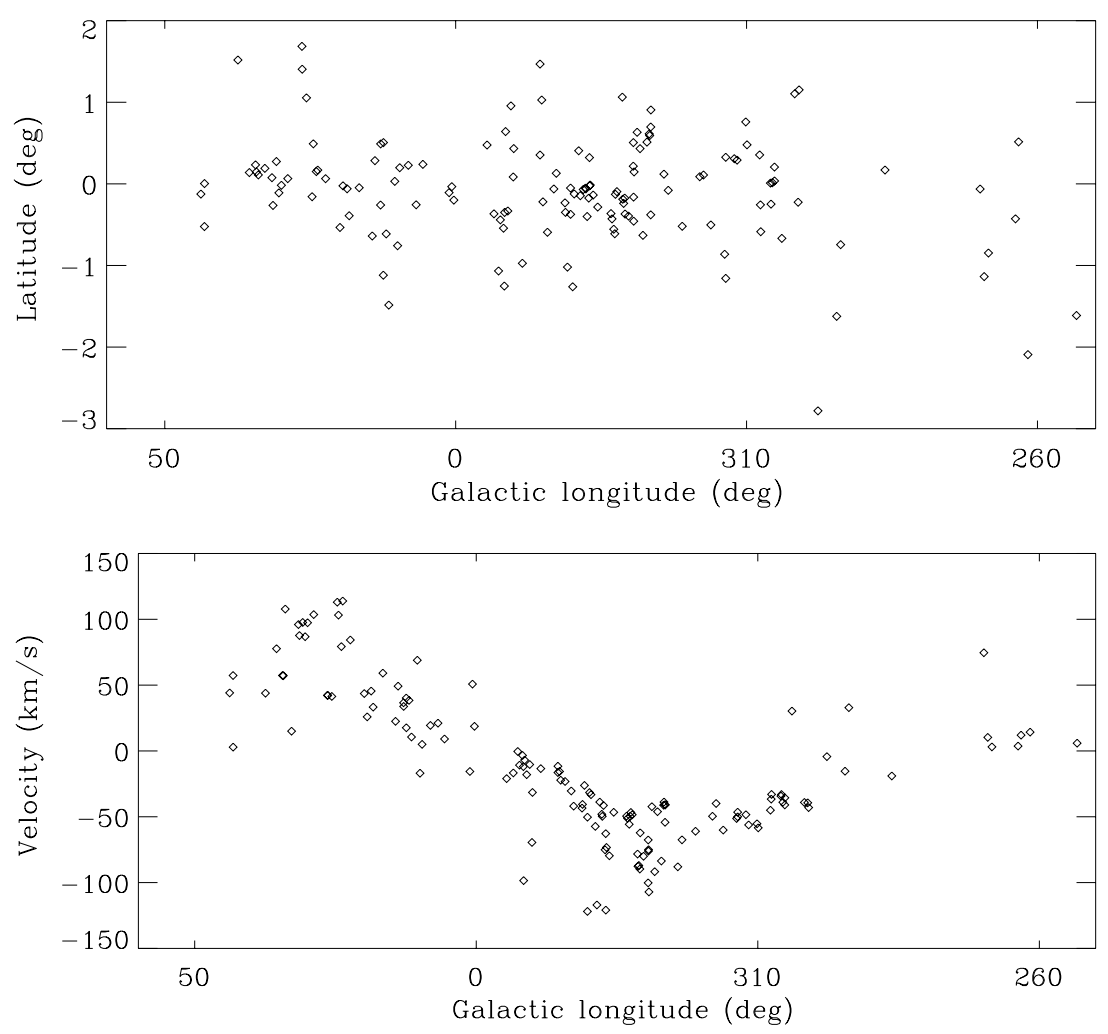

Fig. 1. Galactic distribution of the sources in the survey. Top: longitude-latitude coverage. Bottom: longitude-velocity distribution.

where $k_{v}$ and $D$ are, respectively, the dust mass absorption coefficient and the distance to the source.

The value of the opacity is set by the characteristics of the dust, which are not well determined observationally. Different assumptions about these characteristics can lead to values that differ up to one order of magnitude. The opacities compiled by Hildebrand (1983) have been commonly used in the literature (e.g. Walker et al. 1990; Beuther et al. 2002). More recently, Ossenkopf \& Henning (1994) computed and tabulated the opacity of dust in a broader wavelength range. We adopt a dust opacity at $1.2 \mathrm{~mm}$ of $1 \mathrm{~cm}^{2} \mathrm{~g}^{-1}$, computed by the last authors for typical conditions of dense protostellar clouds. This opacity is a factor of 5 greater than the opacity given by Hildebrand(1983), implying masses five times smaller than those computed using the Hildebrand's value. Assuming a gasto-dust mass ratio $R=M_{\mathrm{g}} / M_{\mathrm{d}}=100$, the total cloud mass is given in terms of the flux density at $1.2 \mathrm{~mm}$ by,

$M_{\mathrm{c}}=20.689\left(\frac{S}{\mathrm{Jy}}\right)\left(\frac{D}{\mathrm{kpc}}\right)^{2}\left\{\exp \left(\frac{11.998}{T_{\mathrm{d}}}\right)-1\right\} M_{\odot}$.

The masses computed using this expression are given in Col. 9 of Table 1. An histogram of the distribution of masses is shown in the middle left panel of Fig. 3. The average value of the masses is $5.0 \times 10^{3} M_{\odot}$.

The densities were determined from the masses assuming a mean mass per particle of $\mu=2.29 m_{\mathrm{H}}$ and that the sources have spherical morphologies with the radius given in Table 1. The distribution of densities is shown in the bottom right panel of Fig. 3. The average density of our sample is
$n_{\mathrm{H}_{2}}=2.1 \times 10^{5} \mathrm{~cm}^{-3}$. The average surface density, $\Sigma=\frac{M}{\pi R^{2}}$, is $1.1 \pm 1.5 \mathrm{~g} \mathrm{~cm}^{-2}$.

\subsection{Bolometric luminosities}

The bolometric luminosities, listed in Col. 11 of Table1, were calculated by integrating the SED. The distribution of luminosities is shown in the middle right panel of Fig. 3. The average luminosity of $2.3 \times 10^{5} L_{\odot}$ corresponds to that of a O6.5 ZAMS star. The median luminosity is $6.2 \times 10^{4} L_{\odot}$, therefore the source of energy of half of our objects would correspond to stars with spectral type of $\mathrm{O} 8$ or earlier if they are excited by a single star. Our sample does, however, contain a few low luminosity objects such as IRAS 08076-3556 (Persi et al. 1994) and IRAS 11590-6452 (Bourke et al. 1997). Most of our sources $(91 \%)$ have luminosities $\geq 10^{4} L_{\odot}$, which correspond to that of a star B0.5 (Panagia 1973).

\subsection{Morphologies}

The 1.2-mm emission associated with the IRAS sources exhibits a variety of morphologies. Filaments are seen in $24 \%$ of the sources, $23 \%$ show clumpy morphologies and $53 \%$ have regular shapes. The mechanisms of formation of massive and dense cores are still poorly known and the variety of structures seen in the maps illustrates the complexity of the problem. The common appearance of filaments in our maps, as well as in other star forming regions (e.g., Mizuno et al. 1995), suggests that gravitational instabilities in filaments are likely to be an important mechanism of core formation (Larson 1985; 

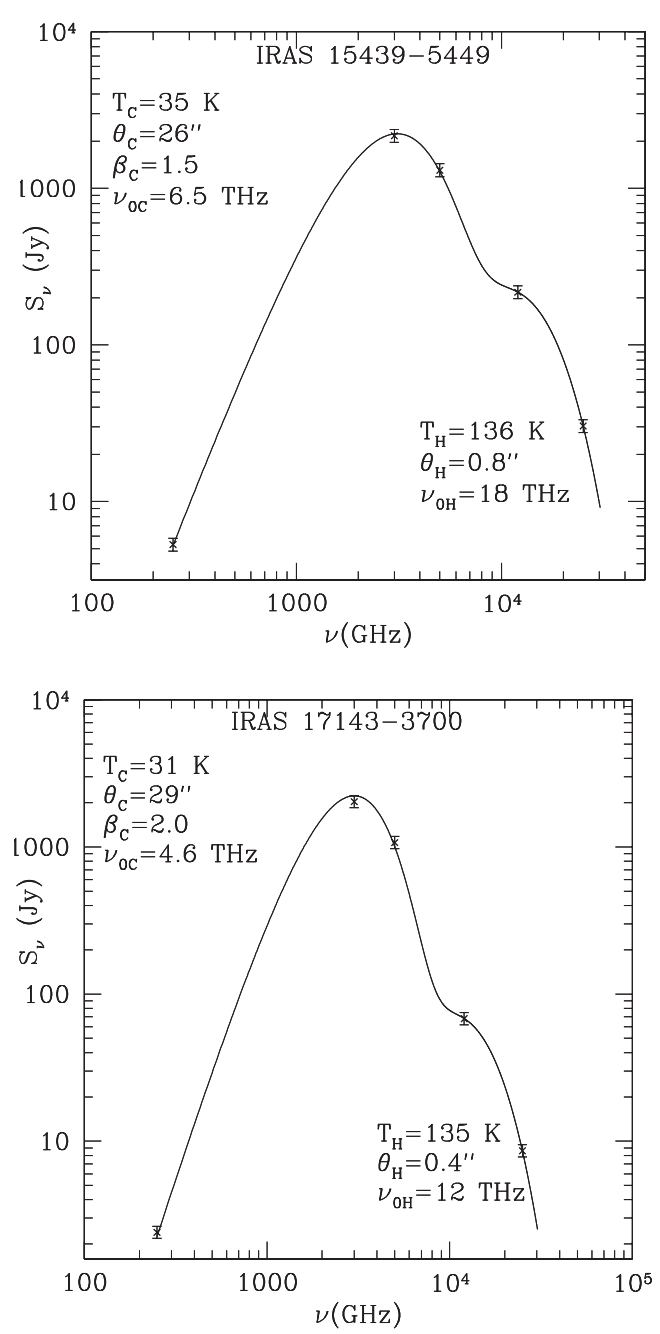

Fig. 2. SED of typical 1.2-mm/IRAS sources. Top: IRAS 15439-5449. Bottom: IRAS 17143-3700. The subscripts C and H refers to cold and hot components, respectively.

Tilley \& Pudritz 2003). The clumpy structure could be explained if the cores are formed by turbulent shocks (Padoan et al. 2001). Therefore, both gravitational fragmentation and turbulent shocks seem to play a fundamental role for the origin of the massive and dense cores. The present survey provides a wealth of observational data to further explore the rol of filamentary structure in massive star formation.

\subsection{The luminosity-to-mass ratio}

Figure 4 shows an histogram of the luminosity-to-mass ratio. The average value of $L / M$ for our sample is $71 \pm 56 L_{\odot} / M_{\odot}$. Comparisons with other works need a scaling of the opacities. Mueller et al. (2002) and Beuther et al. (2002) reported average values of $140 \pm 100$ and $20 \pm 107 L_{\odot} / M_{\odot}$, respectively, which rescaled to our opacity of $1 \mathrm{~cm}^{2} \mathrm{~g}^{-1}$ correspond to values of 133 and $100 L_{\odot} / M_{\odot}$.

Sridharan et al. (2002) proposed that the $L / M$ ratio is an indicator of cloud evolution. To achieve this conclusion, they compared the luminosity-to-mass ratio distribution of sources undetected in radio continuum surveys (Parkes-MIT-NRAO or PMN survey, Griffith et al. 1994, and the 5 GHz Green Bank survey, Gregory \& Condon 1991) with that of sources associated with UC HII regions reported by Hunter (1997) and Hunter et al. (2000). They found that sources with no radio detections have lower values of $L / M$. They suggested that this result can be explained if the embedded cluster becomes more luminous with time. To corroborate this hypothesis we divided our sample into two subsamples, those with and without radio continuum detections in the PMN survey. The right panel of Fig. 4 shows the distribution of the luminosity-to-mass ratio for both subsamples. The average value of $L / M$ for cores associated with UC HII regions is $85 \pm 62$, whereas that of cores not associated with UC HII regions is $57 \pm 45 L_{\odot} / M_{\odot}$. Applying the Kolmogorov-Smirnov test to both subsamples we obtained a K-S statistics of 0.27 with a significance level of 0.007 . Therefore, the null hypothesis (both subsamples are from the same distribution) cannot be discarded.

In the following we describe an alternative approach to explain the difference in the luminosity-to-mass ratio among dust cores assuming that all systems are roughly coeval. Most of the IRAS sources in our sample are luminous and thus are likely to host a cluster of young stars containing a massive star. Due to the strong dependence of luminosity with mass, the total luminosity is dominated by the most luminous member of the cluster. Thus it is possible that the value of the $L / M$ ratio is mainly determined by the most luminous member of the cluster. In what follows we assess this hypothesis in a simple way. Assuming a Salpeter initial mass function $\left(\mathrm{d} N \propto M^{-2.3} \mathrm{~d} M\right)$ and using the mass-to-luminosity relation for stars, $L \propto M^{3.5}$ (Bressan et al. 1993), we obtain that the mass and luminosity of the most massive star of the cluster, $M_{\max }$ and $L_{\max }$ respectively, are related to the total luminosity of the cluster by the expression, $L_{\text {cluster }}=0.3 \times L_{\max } M_{\max }$. Figure 6 plots the $L_{\text {cloud }} / M_{\text {cloud }}$ ratio of the dust cores (determined from the observations) versus $L_{\max }$ (derived using the last expression), showing that there is a clear correlation between these quantities. A robust estimation fit to the observed relationship gives $L_{\text {cloud }} / M_{\text {cloud }}=0.75 L_{\max }^{0.46}$. We conclude that the luminosity-tomass ratio can be interpreted as an indicator of which type of star is the most conspicuous one of the cluster.

\section{Comparison with previous works}

Several surveys of molecular line emission toward massive star-forming regions (hereafter MSFRs) have been carried out during the last decade (see Evans 1999 for a review). The properties of massive and dense cores derived from these observations are in good agreement with those derived by us using as tracer dust emission. For instance, Plume et al. (1997) observed the CS(5 $\rightarrow 4)$ line emission toward several MSFR and found that the cores have an average radius of $0.5 \mathrm{pc}$, and average virial mass of $4 \times 10^{3} M_{\odot}$ and densities, derived from large velocity gradient models, of $8 \times 10^{5} \mathrm{~cm}^{-3}$.

Large surveys of dust emission toward MSFRs have been recently reported by Mueller et al. (2002) and Beuther et al. (2002). We note, beforehand, that since different observational works using as probe the dust emission may assume different opacities, a comparison needs a rescaling of the dust opacities. Mueller et al. (2002) carried out 

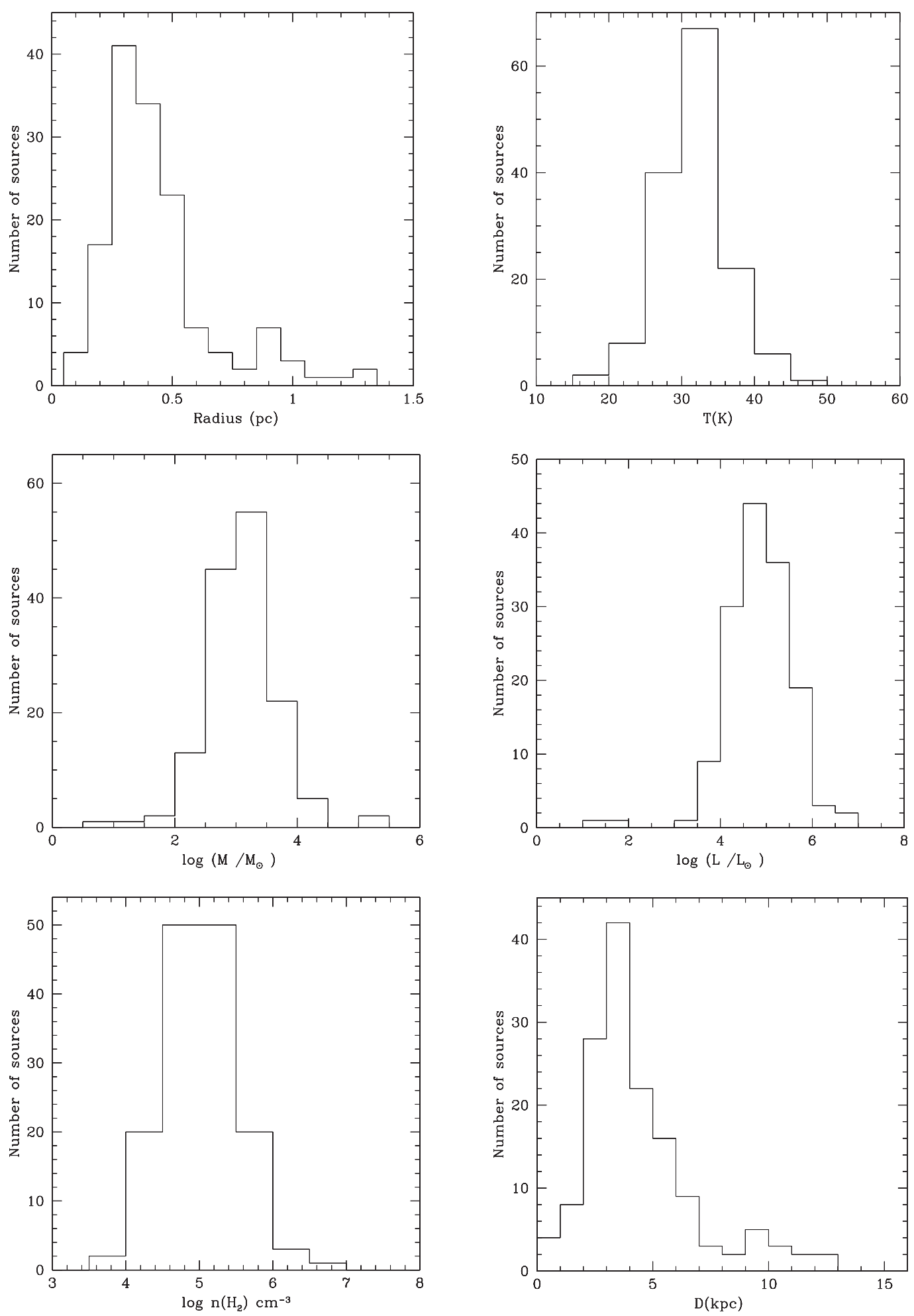

Fig. 3. Distribution of parameters of 1.2-mm cores associated with high-mass star forming regions. Upper left: size distribution. The average radius is $0.4 \pm 0.2 \mathrm{pc}$. Upper right: temperature distribution. The average temperature is $32 \pm 5 \mathrm{~K}$. Middle left: mass distribution. The average mass is $5.0 \times 10^{3} M_{\odot}$. Middle right: luminosity distribution. The average luminosity is $2.3 \times 10^{5} L_{\odot}$. Lower left: density distribution. The average density is $2.1 \times 10^{5} \mathrm{~cm}^{-3}$. Lower right: distance distribution. The average distance is $4.4 \mathrm{kpc}$.

observations of dust emission at submillimeter wavelenghts toward 51 MSFRs associated with water masers. The average luminosity of the sources in their sample is $2.5 \times 10^{5} L_{\odot}$, similar to that of the objects in our sample $\left(1.7 \times 10^{5} L_{\odot}\right)$. They found that the cores in their sample have an average radius of
$0.16 \pm 0.1 \mathrm{pc}$, an average mass of $2.1 \times 10^{3} M_{\odot}$, and an average surface density of $0.77 \pm 1.8 \mathrm{~cm}^{-2}$, in good agreement with our results. Beuther et al. (2002) made observations of dust emission at millimeter wavelengths toward 69 MSFRs selected from Bronfman et al. (1996). The average luminosity of their objects 

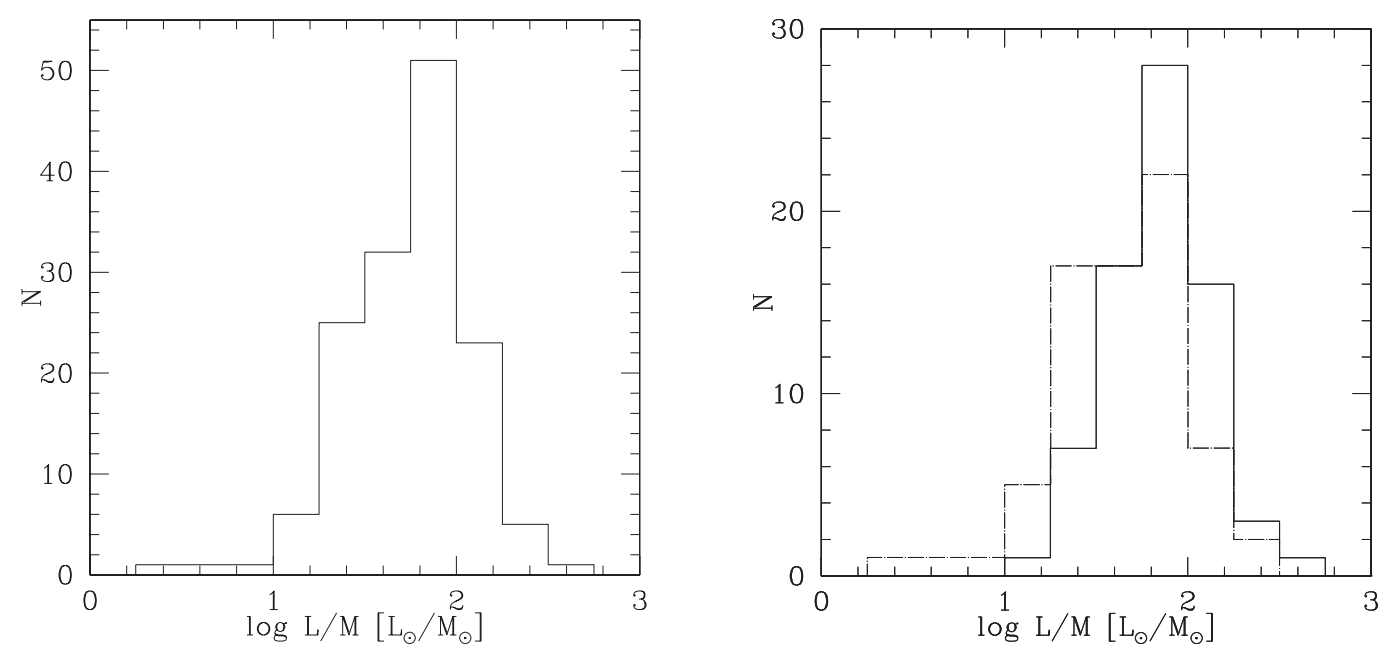

Fig. 4. Left: histogram of the distribution of the $L / M$ ratio for the whole sample of cores. Right: luminosity-to-mass ratio of two subsamples. Dotted line: cores without radio detections; continuous line: cores associated with radio emission. The tail of low $L / M$ in sources with no radio continuum detections is produced by contamination of low-mass cores with non-ionizing sources.

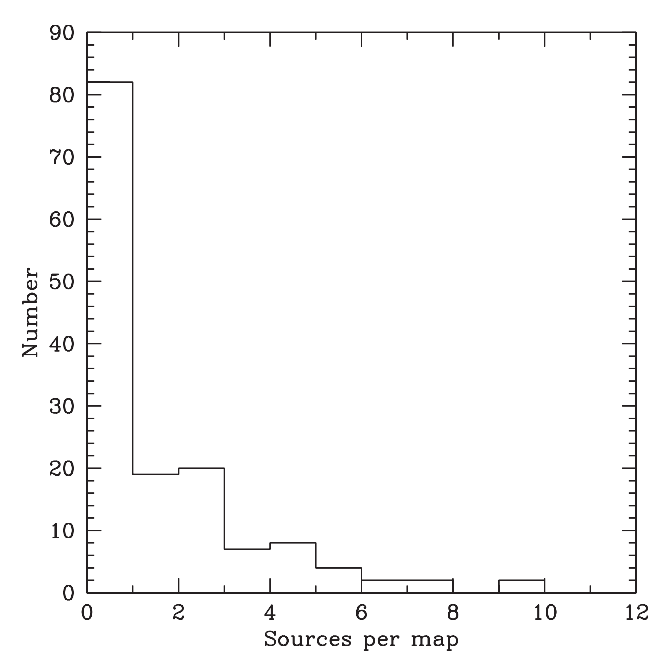

Fig. 5. Histogram of the distribution of the number of sources per map.

is $2.0 \times 10^{4} L_{\odot}$, about ten times smaller than that of the objects in our sample. They found that the cores have an average radius of $0.2 \pm 0.2 \mathrm{pc}$, and (rescaled) average mass of $3.0 \times 10^{2} M_{\odot}$ and average density of $7.8 \times 10^{4} \mathrm{~cm}^{-3}$. Their derived values are about one order of magnitude smaller than the those derived by us, a difference most likely due to the difference in luminosity of the two samples. Walsh et al. (2003) mapped continuum emission at 1200,850 and $450 \mu \mathrm{m}$ toward 71 fields of methanol masers. A comparison of the maps at 1200, 850 and $450 \mu \mathrm{m}$ of the 14 sources in common between this and our survey shows a very good agreement in the morphologies of the dust emission. Further, the flux density dependence with frequency, in the range from $1200 \mu \mathrm{m}$ to $450 \mu \mathrm{m}$, are well fitted with power law relationships of the form $S_{v} \propto v^{\alpha}$, with $\alpha$ being in the range 3 to 4 with an average value of 3.5. This implies a power law index of the optical depth dependence with frequency (see Sect. 3.2) of $\beta \approx 1.5$, similar to those reported by Lis et al. (1998) for Orion clouds.

The dust temperatures of the sources in our sample $\left(\left\langle T_{\mathrm{d}}\right\rangle=\right.$ $32 \pm 5 \mathrm{~K})$ are similar to those derived in other large scale surveys
(Mueller et al. (2002), $\left\langle T_{\mathrm{d}}\right\rangle=29 \pm 9 \mathrm{~K}$; Sridharan et al. (2002), $\left\langle T_{\mathrm{d}}\right\rangle=45 \pm 11$ ) as well as in more limited surveys ( Molinari et al. (2000), $\left\langle T_{\mathrm{d}}\right\rangle=32 \pm 5 \mathrm{~K}$, Hunter (1997), $\left\langle T_{\mathrm{d}}\right\rangle=40 \pm$ $10 \mathrm{~K})$. The derived temperatures are in good agreement with theoretical calculations of temperature profiles of dust cocoons enshrouding UC HII regions (Wolfire \& Churchwell 1994).

\section{Summary}

We have carried out a 1.2-mm continuum emission survey, using the bolometer camera SIMBA at the SEST telescope, toward 146 galactic regions. Each mapped region is about $15^{\prime} \times 10^{\prime}$ in size and is centered on an IRAS source thought to be associated with a region of high-mass star formation. The main results are summarized as follows.

1.2-mm emission was detected toward all IRAS sources. We find that the 1.2-mm/IRAS objects, which are representative of molecular cores harboring recently formed massive stars, have typically sizes of $\sim 0.4 \mathrm{pc}$, masses of $5 \times 10^{3} M_{\odot}$, densities of $2 \times 10^{5} \mathrm{~cm}^{-3}$, dust temperatures of $\sim 32 \mathrm{~K}$, and luminosities of $\sim 2 \times 10^{5} L_{\odot}$.

The $L / M$ ratios of the $1.2-\mathrm{mm} / \mathrm{IRAS}$ sources have an average value of $71 L_{\odot} / M_{\odot}$. We do not find statistically significant differences in the $L / M$ ratios for sources with and without detections in radio continuum surveys. We suggest that the difference in the luminosity-to-mass ratio among the dust cores is related to the luminosity of the most massive star embedded within them rather than being an indicator of the core evolutionary stage.

Acknowledgements. We thank the SEST staff for the help in the observations, especially M. Nielbock for helping with the MOPSI software. S.F. thanks Fundación Andes for financial support. L.B., S.F. and J.M. acknowledge support by FONDECYT Grant 1010431. G.G. acknowledges support from the Chilean Centro de Astrofísica FONDAP 15010003. 


\section{References}

Beuther, H., Schilke, P., Menten, K., et al. 2002, ApJ, 566, 945

Bonnell, I., Bate, M., \& Zinnecker, H. 1998 MNRAS 298, 93

Bourke, T., Garay, G., Lehtinen, K. et al. 1997, ApJ, 476, 781

Bressan, A., Fagotto, F., Bertelli, G., \& Chiosi, C. 1993, A\&AS, 100, 647

Bronfman, L., Nyman, L-A., \& May, J. A. 1996, A\&AS, 115, 81

Bronfman, L., Cassassus S., May, J., \& Nyman L. 2000, A\&A, 521, 534

Chini R., Krugel E., \& Wargau W. 1987, A\&A, 181, 378

Evans, N. J. 1999, ARA\&A 37, 311

Garay, G., \& Lizano S. 1999, PASP, 111, 1049

Garay, G., Brooks, K., Mardones D., \& Norris R. 2003 ApJ, 587, 739

Gregory, P. C., \& Condon, J. J. 1991, ApJS, 75, 1011

Griffith, M., Wright, A., Burke, B., \& Ekers, R. D. 1994, ApJS, 90 , 179

Hatchell, J., Fuller G. A., Millar, T. J., Thompson M. A., \& Macdonald G. H. 2000, A\&A, 357, 637

Hunter, T. 1997, Ph.D. Thesis, Caltech

Hunter, T., Churchwell, E., Watson, C., et al. 2000, ApJ, 119, 2711

Hildebrand, R. 1983, QJRAS, 24, 267

Larson, R. 1985, MNRAS, 214, 379

Lis, D. C., Serabyn, E., Keene, J., et al. 1998, ApJ, 509, 299

McKee, C., \& Tan, J. 2003 ApJ 585, 850

Mezger, P. G. 1994, Ap\&SS, 212, 197

Mizuno, A., Onishi, T., Yonekura, Y., et al. 1995, ApJ, 445, L161
Mueller, K., Shirley, Y., Evans, N., \& Jacobson, H. 2002, ApJS, 143, 469

Molinari, S., Brand, J., Cesaroni, R., \& Palla, F. 2000, A\&A, 355, 617

Nyman, L.-A., Nielbock M., Anciaux M., et al. 2001, Msngr, 106, 40

Ossenkopf, V., \& Henning, Th. 1994, A\&A, 291, 943

Osorio, M., Lizano S., \& D’Alessio P. 1999 ApJ, 525, 808

Padoan, P., Juvela, M., Goodman, A., \& Nordlund, A. 2001, ApJ, 553, 227

Panagia, N. 1973, ApJ 78, 929

Persi, P., Ferrari-Toniolo, M., Marenzi, A. R., et al. 1994, A\&A, 282, 233

Plume, R., Jaffe, D. T., \& Evans, N. J. 1992 ApJS, 78, 505

Plume, R., Jaffe, D. T., Evans, N. J., Martín-Pintado, J., \& Gómez-González, J. 1997 ApJ 476, 730

Shepherd, D., Claussen, M., \& Kurtz, S. 2001 Science, 292, 1513

Sridharan, T. K., Beuther, H., Schilke, P., Menten, M., \& Wyrowski, F. 2002, ApJ, 566, 931

Tilley, D., \& Pudritz, R. 2003, ApJ, 593, 426

Walsh, A. J., Burton, M. G., Hyland, A. R., \& Robinson, G. 1998, MNRAS, 301, 640

Walsh, A. J., Macdonald, G. H., Alvey, N. D. S., Burton, M. G., \& Lee, J.-K. 2003, A\&A, 410, 597

Walker, C., Adams, F., \& Lada, C. 1990 ApJ, 349, 515

Wolfire, M. G., \& Churchwell, E. 1994, ApJ, 427,889

Wood, D. O. S., Churchwell, E. 1989, ApJ, 340, 265

Yorke, H., \& Sonnhalter C. 2002, ApJ, 569, 846

Zylka, R. 1998, Pocket Cookbook for the MOPSI software 


\section{Online Material}


Table 1.

\begin{tabular}{|c|c|c|c|c|c|c|c|c|c|c|}
\hline \multirow[b]{3}{*}{ Source } & \multirow[b]{3}{*}{$\begin{array}{c}D \\
(\mathrm{kpc})\end{array}$} & \multicolumn{5}{|c|}{ Observed parameters } & \multicolumn{4}{|c|}{ Derived parameters } \\
\hline & & \multirow[b]{2}{*}{$N$} & \multicolumn{2}{|c|}{ Peak coordinates } & \multirow[b]{2}{*}{$\begin{array}{c}S_{1.2 \mathrm{~mm}} \\
(\mathrm{Jy})\end{array}$} & \multirow[b]{2}{*}{$\begin{array}{l}\theta_{\mathrm{S}} \\
(")\end{array}$} & \multirow[b]{2}{*}{$\begin{array}{l}T_{\mathrm{d}} \\
(\mathrm{K})\end{array}$} & \multirow[b]{2}{*}{$\begin{array}{c}M_{\mathrm{c}} \\
\left(M_{\odot}\right)\end{array}$} & \multirow[b]{2}{*}{$\begin{array}{c}n_{\mathrm{H}_{2}} \\
\left(\mathrm{~cm}^{-3}\right)\end{array}$} & \multirow[b]{2}{*}{$\begin{array}{c}L \\
\left(L_{\odot}\right)\end{array}$} \\
\hline & & & $\begin{array}{c}\alpha \\
(1950)\end{array}$ & $\begin{array}{c}\delta \\
(1950)\end{array}$ & & & & & & \\
\hline 08076-3556 & 0.4 & 1 & 080739.78 & -355619.8 & 1.8 & 37 & 18 & $5.6 \times 10^{0}$ & $4.0 \times 10^{5}$ & $1.6 \times 10^{1}$ \\
\hline 08303-4303 & 2.3 & 1 & 083024.08 & -430339.4 & 4.8 & 29 & 30 & $2.6 \times 10^{2}$ & $1.9 \times 10^{5}$ & $6.7 \times 10^{3}$ \\
\hline $08448-4343$ & 0.7 & 1 & 084447.25 & -434332.8 & 6.1 & 44 & 25 & $3.9 \times 10^{1}$ & $2.9 \times 10^{5}$ & $1.1 \times 10^{3}$ \\
\hline $08470-4243$ & 2.1 & 1 & 084659.36 & -424320.6 & 5.8 & 31 & 33 & $2.3 \times 10^{2}$ & $1.8 \times 10^{5}$ & $1.1 \times 10^{4}$ \\
\hline $09002-4732$ & 1.2 & 1 & 090012.07 & -473208.7 & 24.0 & 41 & 39 & $2.6 \times 10^{2}$ & $4.9 \times 10^{5}$ & $3.9 \times 10^{4}$ \\
\hline 09018-4816 & 2.6 & 4 & 090151.64 & -481612.9 & 14.7 & 35 & 31 & $9.8 \times 10^{2}$ & $2.9 \times 10^{5}$ & $5.2 \times 10^{4}$ \\
\hline 09094-4803 & 9.6 & 2 & 090923.91 & -480328.9 & 1.1 & 30 & 23 & $1.4 \times 10^{3}$ & $1.3 \times 10^{4}$ & $4.0 \times 10^{4}$ \\
\hline $10365-5803$ & 2.4 & 1 & 103636.21 & -580336.8 & 8.0 & 38 & 30 & $4.7 \times 10^{2}$ & $1.4 \times 10^{5}$ & $1.9 \times 10^{4}$ \\
\hline $11298-6155$ & 10.0 & 1 & 112946.34 & -615556.3 & 3.0 & 28 & 32 & $2.7 \times 10^{3}$ & $2.7 \times 10^{4}$ & $1.7 \times 10^{5}$ \\
\hline $11332-6258$ & 1.9 & 1 & 113311.33 & -625812.2 & 3.3 & 30 & 30 & $1.3 \times 10^{2}$ & $1.4 \times 10^{5}$ & $5.3 \times 10^{3}$ \\
\hline $11590-6452$ & 0.4 & 1 & 115900.61 & -645210.3 & 5.4 & 30 & 22 & $1.3 \times 10^{1}$ & $1.5 \times 10^{6}$ & $5.3 \times 10^{1}$ \\
\hline $12320-6122$ & 4.4 & 2 & 123200.06 & -612320.1 & 13.8 & 35 & 37 & $2.1 \times 10^{3}$ & $1.3 \times 10^{5}$ & $2.2 \times 10^{5}$ \\
\hline $12326-6245$ & 4.4 & 1 & 123240.75 & -624605.9 & 17.3 & 31 & 40 & $2.4 \times 10^{3}$ & $2.2 \times 10^{5}$ & $2.7 \times 10^{5}$ \\
\hline $12383-6128$ & 4.5 & 2 & 123823.58 & -612815.9 & 6.7 & 58 & 27 & $1.6 \times 10^{3}$ & $2.1 \times 10^{4}$ & $4.9 \times 10^{4}$ \\
\hline $12572-6316$ & 2.9 & 1 & 125718.05 & -631622.2 & 3.0 & 35 & 29 & $2.7 \times 10^{2}$ & $5.8 \times 10^{4}$ & $5.6 \times 10^{3}$ \\
\hline $13079-6218$ & 4.9 & 5 & 130800.31 & -621842.2 & 18.5 & 35 & 36 & $3.6 \times 10^{3}$ & $1.6 \times 10^{5}$ & $2.8 \times 10^{5}$ \\
\hline $13080-6229$ & 3.4 & 6 & 130800.60 & -622903.1 & 8.3 & 35 & 39 & $7.4 \times 10^{2}$ & $9.0 \times 10^{4}$ & $3.2 \times 10^{5}$ \\
\hline $13111-6228$ & 4.0 & 7 & 131111.13 & -622828.0 & 11.1 & 42 & 32 & $1.6 \times 10^{3}$ & $7.6 \times 10^{4}$ & $1.3 \times 10^{5}$ \\
\hline $13134-6242$ & 3.0 & 2 & 131326.22 & -624239.7 & 6.7 & 26 & 35 & $5.1 \times 10^{2}$ & $2.4 \times 10^{5}$ & $3.1 \times 10^{4}$ \\
\hline $13140-6226$ & 3.1 & 1 & 131400.81 & -622635.4 & 2.9 & 30 & 28 & $3.1 \times 10^{2}$ & $7.9 \times 10^{4}$ & $7.8 \times 10^{3}$ \\
\hline $13291-6229$ & 3.2 & 1 & 132909.66 & -622955.0 & 3.5 & 45 & 30 & $3.5 \times 10^{2}$ & $2.7 \times 10^{4}$ & $4.4 \times 10^{4}$ \\
\hline $13291-6249$ & 2.8 & 1 & 132906.10 & -624952.2 & 4.8 & 29 & 32 & $3.6 \times 10^{2}$ & $1.4 \times 10^{5}$ & $2.5 \times 10^{4}$ \\
\hline $13295-6152$ & 4.5 & 1 & 132931.17 & -615216.2 & 2.7 & 39 & 25 & $7.2 \times 10^{2}$ & $2.9 \times 10^{4}$ & $7.0 \times 10^{3}$ \\
\hline $13471-6120$ & 5.4 & 3 & 134710.64 & -612025.3 & 9.1 & 30 & 39 & $2.0 \times 10^{3}$ & $1.0 \times 10^{5}$ & $2.7 \times 10^{5}$ \\
\hline $13484-6100$ & 5.5 & 1 & 134827.81 & -610049.7 & 4.0 & 28 & 32 & $1.1 \times 10^{3}$ & $6.9 \times 10^{4}$ & $7.1 \times 10^{4}$ \\
\hline $14013-6105$ & 5.6 & 4 & 140118.11 & -610543.5 & 7.0 & 31 & 35 & $1.9 \times 10^{3}$ & $7.5 \times 10^{4}$ & $1.7 \times 10^{5}$ \\
\hline $14050-6056$ & 3.9 & 2 & 140503.91 & -605634.7 & 5.8 & 35 & 34 & $7.7 \times 10^{2}$ & $6.7 \times 10^{4}$ & $7.2 \times 10^{4}$ \\
\hline $14164-6028$ & 3.5 & 1 & 141626.37 & -602824.9 & 1.0 & 27 & 29 & $1.3 \times 10^{2}$ & $3.4 \times 10^{4}$ & $9.3 \times 10^{3}$ \\
\hline $14206-6151$ & 3.9 & 1 & 142034.86 & -615158.6 & 2.8 & 35 & 27 & $4.9 \times 10^{2}$ & $4.4 \times 10^{4}$ & $1.3 \times 10^{4}$ \\
\hline $14212-6131$ & 4.0 & 1 & 142116.74 & -613129.8 & 7.3 & 37 & 27 & $1.3 \times 10^{3}$ & $9.7 \times 10^{4}$ & $1.2 \times 10^{5}$ \\
\hline $14382-6017$ & 4.5 & 1 & 143812.20 & -601742.0 & 4.4 & 42 & 29 & $8.4 \times 10^{2}$ & $2.6 \times 10^{4}$ & $7.5 \times 10^{4}$ \\
\hline $14453-5912$ & 2.8 & 2 & 144518.61 & -591216.9 & 5.3 & 39 & 30 & $4.2 \times 10^{2}$ & $7.2 \times 10^{4}$ & $2.8 \times 10^{4}$ \\
\hline $14498-5856$ & 3.5 & 1 & 144952.47 & -585640.1 & 7.1 & 33 & 30 & $8.7 \times 10^{2}$ & $1.3 \times 10^{5}$ & $3.5 \times 10^{4}$ \\
\hline $15122-5801$ & 4.1 & 3 & 151214.07 & -580035.8 & 4.4 & 36 & 31 & $7.3 \times 10^{2}$ & $5.1 \times 10^{4}$ & $7.2 \times 10^{4}$ \\
\hline $15254-5621$ & 4.4 & 1 & 152527.34 & -562110.0 & 11.6 & 34 & 35 & $1.9 \times 10^{3}$ & $1.2 \times 10^{5}$ & $1.2 \times 10^{5}$ \\
\hline $15290-5546$ & 6.0 & 1 & 152900.60 & -554602.9 & 12.8 & 33 & 36 & $3.7 \times 10^{3}$ & $1.0 \times 10^{5}$ & $3.9 \times 10^{5}$ \\
\hline $15384-5348$ & 2.8 & 3 & 153827.65 & -534858.1 & 16.4 & 55 & 34 & $1.1 \times 10^{3}$ & $6.8 \times 10^{4}$ & $1.0 \times 10^{5}$ \\
\hline $15394-5358$ & 2.8 & 1 & 153927.97 & -535748.3 & 17.2 & 33 & 25 & $1.8 \times 10^{3}$ & $4.8 \times 10^{5}$ & $1.5 \times 10^{4}$ \\
\hline $15408-5356$ & 2.7 & 5 & 154053.63 & -535625.6 & 28.2 & 46 & 37 & $1.6 \times 10^{3}$ & $1.9 \times 10^{5}$ & $1.9 \times 10^{5}$ \\
\hline
\end{tabular}


Table 1. continued.

\begin{tabular}{|c|c|c|c|c|c|c|c|c|c|c|}
\hline \multirow[b]{3}{*}{ Source } & \multirow[b]{3}{*}{$\begin{array}{c}D \\
(\mathrm{kpc})\end{array}$} & \multicolumn{5}{|c|}{ Observed parameters } & \multicolumn{4}{|c|}{ Derived parameters } \\
\hline & & \multirow[b]{2}{*}{$N$} & \multicolumn{2}{|c|}{ Peak coordinates } & \multirow[b]{2}{*}{$\begin{array}{c}S_{1.2 \mathrm{~mm}} \\
(\mathrm{Jy})\end{array}$} & \multirow[b]{2}{*}{$\begin{array}{l}\theta_{\mathrm{S}} \\
(")\end{array}$} & \multirow[b]{2}{*}{$\begin{array}{l}T_{\mathrm{d}} \\
(\mathrm{K})\end{array}$} & \multirow[b]{2}{*}{$\begin{array}{c}M_{\mathrm{c}} \\
\left(M_{\odot}\right)\end{array}$} & \multirow[b]{2}{*}{$\begin{array}{c}n_{\mathrm{H}_{2}} \\
\left(\mathrm{~cm}^{-3}\right)\end{array}$} & \multirow[b]{2}{*}{$\begin{array}{c}L \\
\left(L_{\odot}\right)\end{array}$} \\
\hline & & & $\begin{array}{c}\alpha \\
(1950)\end{array}$ & $\begin{array}{c}\delta \\
(1950)\end{array}$ & & & & & & \\
\hline $15411-5352$ & 2.8 & 5 & 154109.25 & -535250.8 & 11.5 & 34 & 35 & $7.7 \times 10^{2}$ & $1.9 \times 10^{5}$ & $1.4 \times 10^{5}$ \\
\hline $15437-5343$ & 5.2 & 1 & 154341.92 & -534336.4 & 3.3 & 31 & 34 & $7.9 \times 10^{2}$ & $4.3 \times 10^{4}$ & $6.1 \times 10^{4}$ \\
\hline $15439-5449$ & 3.5 & 1 & 154356.20 & -544923.2 & 5.3 & 32 & 35 & $5.7 \times 10^{2}$ & $8.7 \times 10^{4}$ & $4.4 \times 10^{4}$ \\
\hline $15502-5302$ & 5.6 & 1 & 155016.36 & -530253.0 & 17.0 & 29 & 45 & $3.4 \times 10^{3}$ & $1.7 \times 10^{5}$ & $7.5 \times 10^{5}$ \\
\hline $15520-5234$ & 2.9 & 1 & 155200.02 & -523427.1 & 20.3 & 30 & 44 & $1.1 \times 10^{3}$ & $3.7 \times 10^{5}$ & $2.0 \times 10^{5}$ \\
\hline $15522-5411$ & 3.1 & 2 & 155215.34 & -541110.2 & 4.8 & 50 & 29 & $5.0 \times 10^{2}$ & $2.9 \times 10^{4}$ & $2.1 \times 10^{4}$ \\
\hline $15557-5215$ & 4.2 & 3 & 155551.68 & -521502.5 & 8.3 & 33 & 27 & $1.7 \times 10^{3}$ & $1.4 \times 10^{5}$ & $2.3 \times 10^{4}$ \\
\hline $15567-5236$ & 7.0 & 6 & 155644.76 & -523627.1 & 20.7 & 30 & 40 & $7.2 \times 10^{3}$ & $1.7 \times 10^{5}$ & $8.0 \times 10^{5}$ \\
\hline $15570-5227$ & 6.1 & 2 & 155705.58 & -522801.3 & 5.6 & 36 & 34 & $1.8 \times 10^{3}$ & $3.7 \times 10^{4}$ & $1.6 \times 10^{5}$ \\
\hline $15584-5247$ & 4.7 & 1 & 155829.00 & -524702.2 & 4.8 & 43 & 31 & $1.1 \times 10^{3}$ & $2.8 \times 10^{4}$ & $3.6 \times 10^{4}$ \\
\hline $15596-5301$ & 4.6 & 1 & 155940.39 & $\begin{array}{lll}-53 & 01 & 17.2\end{array}$ & 5.9 & 33 & 30 & $1.3 \times 10^{3}$ & $7.7 \times 10^{4}$ & $6.4 \times 10^{4}$ \\
\hline $16026-5035$ & 4.8 & 4 & 160240.24 & -503516.9 & 5.9 & 51 & 31 & $1.3 \times 10^{3}$ & $2.1 \times 10^{4}$ & $1.4 \times 10^{5}$ \\
\hline $16037-5223$ & 4.9 & 1 & 160347.95 & -522252.0 & 8.9 & 29 & 34 & $1.9 \times 10^{3}$ & $1.4 \times 10^{5}$ & $1.1 \times 10^{5}$ \\
\hline $16060-5146$ & 5.3 & 2 & 160602.50 & -514716.1 & 38.3 & 28 & 46 & $6.8 \times 10^{3}$ & $4.5 \times 10^{5}$ & $7.5 \times 10^{5}$ \\
\hline $16065-5158$ & 4.0 & 2 & 160630.53 & -515824.2 & 23.8 & 28 & 40 & $2.7 \times 10^{3}$ & $4.4 \times 10^{5}$ & $2.9 \times 10^{5}$ \\
\hline $16071-5142$ & 5.2 & 1 & 160710.27 & -514234.5 & 12.8 & 31 & 29 & $3.7 \times 10^{3}$ & $1.9 \times 10^{5}$ & $8.3 \times 10^{4}$ \\
\hline $16076-5134$ & 5.2 & 1 & 160738.23 & -513415.8 & 10.7 & 38 & 33 & $2.6 \times 10^{3}$ & $7.6 \times 10^{4}$ & $2.0 \times 10^{5}$ \\
\hline $16119-5048$ & 3.3 & 3 & 161157.73 & -504821.2 & 7.3 & 32 & 30 & $8.2 \times 10^{2}$ & $1.6 \times 10^{5}$ & $3.3 \times 10^{4}$ \\
\hline $16132-5039$ & 3.2 & 6 & 161313.39 & -503932.5 & 6.7 & 43 & 33 & $6.3 \times 10^{2}$ & $5.2 \times 10^{4}$ & $6.3 \times 10^{4}$ \\
\hline $16158-5055$ & 3.4 & 5 & 161549.79 & -505600.9 & 21.4 & 53 & 35 & $2.0 \times 10^{3}$ & $7.8 \times 10^{4}$ & $1.6 \times 10^{5}$ \\
\hline $16164-5046$ & 3.7 & 4 & 161623.20 & -504557.7 & 35.2 & 27 & 44 & $3.2 \times 10^{3}$ & $7.0 \times 10^{5}$ & $4.0 \times 10^{5}$ \\
\hline $16172-5028$ & 3.5 & 7 & 161714.63 & -502826.4 & 67.3 & 70 & 33 & $7.4 \times 10^{3}$ & $1.1 \times 10^{5}$ & $4.4 \times 10^{5}$ \\
\hline $16177-5018$ & 3.4 & 4 & 161745.65 & -501813.0 & 46.0 & 32 & 39 & $4.0 \times 10^{3}$ & $7.2 \times 10^{5}$ & $3.4 \times 10^{5}$ \\
\hline $16272-4837$ & 3.4 & 1 & 162714.53 & -483718.8 & 9.3 & 31 & 27 & $1.2 \times 10^{3}$ & $2.3 \times 10^{5}$ & $3.0 \times 10^{4}$ \\
\hline $16297-4757$ & 10.7 & 2 & 162946.85 & -475727.7 & 6.3 & 34 & 32 & $6.6 \times 10^{3}$ & $3.0 \times 10^{4}$ & $5.1 \times 10^{5}$ \\
\hline $16304-4710$ & 11.4 & 1 & 163022.74 & -471024.3 & 1.8 & 33 & 33 & $2.2 \times 10^{3}$ & $8.7 \times 10^{3}$ & $1.0 \times 10^{5}$ \\
\hline $16313-4729$ & 11.0 & 4 & 163113.21 & -472936.8 & 8.1 & 43 & 31 & $9.3 \times 10^{3}$ & $2.0 \times 10^{4}$ & $9.3 \times 10^{5}$ \\
\hline $16318-4724$ & 6.6 & 4 & 163152.44 & -472455.2 & 8.2 & 28 & 33 & $3.2 \times 10^{3}$ & $1.1 \times 10^{5}$ & $2.5 \times 10^{5}$ \\
\hline $16330-4725$ & 10.9 & 1 & 163301.85 & -472521.2 & 9.3 & 49 & 39 & $8.2 \times 10^{3}$ & $1.2 \times 10^{4}$ & $1.9 \times 10^{6}$ \\
\hline $16344-4658$ & 3.6 & 3 & 163428.47 & -465904.4 & 9.0 & 42 & 27 & $1.3 \times 10^{3}$ & $8.5 \times 10^{4}$ & $5.1 \times 10^{4}$ \\
\hline $16348-4654$ & 12.2 & 7 & 163449.27 & -465441.4 & 12.1 & 28 & 30 & $1.8 \times 10^{4}$ & $9.9 \times 10^{4}$ & $6.5 \times 10^{5}$ \\
\hline $16351-4722$ & 3.2 & 1 & 163508.03 & -472208.7 & 10.7 & 29 & 36 & $8.6 \times 10^{2}$ & $2.5 \times 10^{5}$ & $9.3 \times 10^{4}$ \\
\hline $16362-4639$ & 3.1 & 3 & 163617.01 & -463924.2 & 1.8 & 48 & 24 & $2.3 \times 10^{2}$ & $1.6 \times 10^{4}$ & $1.7 \times 10^{4}$ \\
\hline $16372-4545$ & 4.1 & 2 & 163716.37 & -454513.1 & 2.2 & 39 & 25 & $4.6 \times 10^{2}$ & $2.6 \times 10^{4}$ & $3.7 \times 10^{4}$ \\
\hline $16385-4619$ & 6.4 & 1 & 163833.87 & -461948.0 & 4.5 & 29 & 33 & $1.7 \times 10^{3}$ & $5.6 \times 10^{4}$ & $1.3 \times 10^{5}$ \\
\hline $16424-4531$ & 2.8 & 2 & 164227.89 & -453132.7 & 7.8 & 41 & 30 & $6.4 \times 10^{2}$ & $9.4 \times 10^{4}$ & $1.9 \times 10^{4}$ \\
\hline $16445-4459$ & 6.5 & 1 & 164427.60 & -445954.7 & 4.4 & 30 & 31 & $1.8 \times 10^{3}$ & $5.2 \times 10^{4}$ & $1.1 \times 10^{5}$ \\
\hline $16458-4512$ & 3.8 & 1 & 164552.31 & -451248.8 & 14.2 & 53 & 29 & $2.2 \times 10^{3}$ & $5.9 \times 10^{4}$ & $5.1 \times 10^{4}$ \\
\hline $16484-4603$ & 2.7 & 1 & 164823.75 & -460326.6 & 10.1 & 40 & 35 & $6.6 \times 10^{2}$ & $1.1 \times 10^{5}$ & $5.5 \times 10^{4}$ \\
\hline
\end{tabular}


Table 1. continued.

\begin{tabular}{|c|c|c|c|c|c|c|c|c|c|c|}
\hline \multirow[b]{3}{*}{ Source } & \multirow[b]{3}{*}{$\begin{array}{c}D \\
(\mathrm{kpc})\end{array}$} & \multicolumn{5}{|c|}{ Observed parameters } & \multicolumn{4}{|c|}{ Derived parameters } \\
\hline & & \multirow[b]{2}{*}{$N$} & \multicolumn{2}{|c|}{ Peak coordinates } & \multirow[b]{2}{*}{$\begin{array}{c}S_{1.2 \mathrm{~mm}} \\
(\mathrm{Jy})\end{array}$} & \multirow[b]{2}{*}{$\begin{array}{l}\theta_{\mathrm{S}} \\
\text { (") }\end{array}$} & \multirow[b]{2}{*}{$\begin{array}{c}T_{\mathrm{d}} \\
(\mathrm{K})\end{array}$} & \multirow[b]{2}{*}{$\begin{array}{c}M_{\mathrm{c}} \\
\left(M_{\odot}\right)\end{array}$} & \multirow[b]{2}{*}{$\begin{array}{c}n_{\mathrm{H}_{2}} \\
\left(\mathrm{~cm}^{-3}\right)\end{array}$} & \multirow[b]{2}{*}{$\begin{array}{c}L \\
\left(L_{\odot}\right)\end{array}$} \\
\hline & & & $\begin{array}{c}\alpha \\
(1950)\end{array}$ & $\begin{array}{c}\delta \\
(1950)\end{array}$ & & & & & & \\
\hline $16487-4423$ & 3.5 & 4 & 164846.65 & -442245.7 & 5.6 & 44 & 30 & $7.0 \times 10^{2}$ & $4.1 \times 10^{4}$ & $3.0 \times 10^{4}$ \\
\hline $16489-4431$ & 3.4 & 1 & 164856.89 & -443121.7 & 3.8 & 35 & 28 & $4.7 \times 10^{2}$ & $6.3 \times 10^{4}$ & $1.5 \times 10^{4}$ \\
\hline $16506-4512$ & 2.4 & 3 & 165038.32 & -451251.9 & 26 & 63 & 33 & $1.4 \times 10^{3}$ & $8.5 \times 10^{4}$ & $1.1 \times 10^{5}$ \\
\hline $16524-4300$ & 3.6 & 1 & 165228.95 & $\begin{array}{lll}-43 & 00 & 10.2\end{array}$ & 10.3 & 33 & 31 & $1.3 \times 10^{3}$ & $1.7 \times 10^{5}$ & $4.2 \times 10^{4}$ \\
\hline $16547-4247$ & 2.9 & 1 & 165443.66 & -424732.2 & 17.6 & 33 & 33 & $1.4 \times 10^{3}$ & $3.3 \times 10^{5}$ & $6.3 \times 10^{4}$ \\
\hline $16562-3959$ & 1.5 & 10 & 165614.72 & -395925.9 & 81.3 & 43 & 38 & $1.5 \times 10^{3}$ & $1.2 \times 10^{6}$ & $4.8 \times 10^{4}$ \\
\hline $16571-4029$ & 1.9 & 8 & 165703.00 & -402957.5 & 17 & 68 & 37 & $5.0 \times 10^{2}$ & $7.7 \times 10^{5}$ & $7.6 \times 10^{4}$ \\
\hline $17006-4215$ & 2.6 & 2 & 170040.23 & -421547.2 & 11.5 & 29 & 33 & $6.7 \times 10^{2}$ & $3.7 \times 10^{5}$ & $4.1 \times 10^{4}$ \\
\hline $17008-4040$ & 2.0 & 2 & 170052.90 & -404021.7 & 38.6 & 38 & 31 & $1.5 \times 10^{3}$ & $7.9 \times 10^{5}$ & $5.2 \times 10^{4}$ \\
\hline $17016-4124$ & 2.6 & 2 & 170140.21 & -412514.5 & 27.1 & 31 & 32 & $1.7 \times 10^{3}$ & $7.5 \times 10^{5}$ & $5.9 \times 10^{4}$ \\
\hline $17136-3617$ & 1.9 & 1 & 171340.14 & -361743.3 & 20.0 & 37 & 31 & $7.2 \times 10^{2}$ & $4.5 \times 10^{5}$ & $1.1 \times 10^{5}$ \\
\hline $17143-3700$ & 4.0 & 1 & 171422.28 & -365956.9 & 2.4 & 29 & 31 & $3.7 \times 10^{2}$ & $5.3 \times 10^{4}$ & $4.1 \times 10^{4}$ \\
\hline $17158-3901$ & 2.0 & 3 & 171553.69 & -390051.4 & 18.4 & 31 & 32 & $7.2 \times 10^{2}$ & $4.4 \times 10^{5}$ & $2.2 \times 10^{4}$ \\
\hline $17160-3707$ & 5.8 & 4 & 171603.07 & -370748.1 & 37.2 & 63 & 30 & $1.3 \times 10^{4}$ & $5.8 \times 10^{4}$ & $4.3 \times 10^{5}$ \\
\hline $17175-3544$ & 1.5 & 4 & 171732.42 & -354403.1 & 50.0 & 29 & 43 & $8.1 \times 10^{2}$ & $1.9 \times 10^{6}$ & $7.8 \times 10^{4}$ \\
\hline $17204-3636$ & 3.0 & 1 & 172028.16 & -363618.6 & 6.6 & 36 & 30 & $6.3 \times 10^{2}$ & $1.0 \times 10^{5}$ & $1.5 \times 10^{4}$ \\
\hline $17220-3609$ & 10.0 & 1 & 172203.27 & -361003.5 & 23.4 & 34 & 30 & $2.4 \times 10^{4}$ & $1.4 \times 10^{5}$ & $6.8 \times 10^{5}$ \\
\hline $17233-3606$ & 0.8 & 3 & 172321.05 & -360643.2 & 54.0 & 28 & 45 & $2.1 \times 10^{2}$ & $3.8 \times 10^{6}$ & $1.7 \times 10^{4}$ \\
\hline $17244-3536$ & 2.4 & 1 & 172428.09 & -353651.3 & 5.0 & 37 & 31 & $2.8 \times 10^{2}$ & $9.1 \times 10^{4}$ & $1.9 \times 10^{4}$ \\
\hline $17258-3637$ & 2.4 & 3 & 172555.10 & -363759.8 & 37.0 & 32 & 42 & $1.4 \times 10^{3}$ & $7.5 \times 10^{5}$ & $1.9 \times 10^{5}$ \\
\hline $17269-3312$ & 4.2 & 5 & 172657.72 & -331231.8 & 4.0 & 35 & 31 & $7.0 \times 10^{2}$ & $1.1 \times 10^{5}$ & $4.9 \times 10^{4}$ \\
\hline $17271-3439$ & 3.8 & 1 & 172706.85 & -343923.7 & 59.2 & 39 & 35 & $7.1 \times 10^{3}$ & $4.9 \times 10^{5}$ & $2.6 \times 10^{5}$ \\
\hline $17278-3541$ & 1.2 & 2 & 172753.58 & -354215.5 & 17.2 & 66 & 27 & $2.8 \times 10^{2}$ & $1.2 \times 10^{5}$ & $5.8 \times 10^{3}$ \\
\hline $17439-2845$ & 8.0 & 1 & 174359.47 & -284455.3 & 9.3 & 46 & 30 & $6.1 \times 10^{3}$ & $2.7 \times 10^{4}$ & $4.8 \times 10^{5}$ \\
\hline $17441-2822$ & 8.1 & 2 & 174409.37 & -282203.4 & 447 & 64 & 35 & $2.5 \times 10^{5}$ & $4.0 \times 10^{5}$ & $3.7 \times 10^{6}$ \\
\hline $17455-2800$ & 10 & 1 & 174532.46 & -280044.1 & 13.8 & 45 & 31 & $1.3 \times 10^{4}$ & $3.1 \times 10^{4}$ & $8.1 \times 10^{5}$ \\
\hline $17545-2357$ & 2.5 & 1 & 175430.26 & -235748.2 & 7.6 & 48 & 30 & $4.8 \times 10^{2}$ & $6.0 \times 10^{4}$ & $1.4 \times 10^{4}$ \\
\hline $17589-2312$ & 3.8 & 1 & 175855.01 & -231219.3 & 1.4 & 29 & 30 & $2.1 \times 10^{2}$ & $3.4 \times 10^{4}$ & $2.1 \times 10^{4}$ \\
\hline $17599-2148$ & 3.4 & 1 & 180000.80 & -214811.6 & 18.2 & 41 & 32 & $1.9 \times 10^{3}$ & $1.7 \times 10^{5}$ & $1.1 \times 10^{5}$ \\
\hline $18032-2032$ & 5.7 & 1 & 180315.76 & -203156.4 & 11.4 & 29 & 38 & $3.0 \times 10^{3}$ & $1.5 \times 10^{5}$ & $3.2 \times 10^{5}$ \\
\hline 18056-1952 & 5.6 & 1 & 180540.46 & -195216.5 & 15.2 & 33 & 34 & $4.2 \times 10^{3}$ & $1.5 \times 10^{5}$ & $3.5 \times 10^{5}$ \\
\hline $18075-2040$ & 5.0 & 3 & 180735.97 & -204000.2 & 1.7 & 39 & 21 & $6.8 \times 10^{2}$ & $2.1 \times 10^{4}$ & $2.4 \times 10^{4}$ \\
\hline $18079-1756$ & 2.4 & 1 & 180755.68 & -175621.5 & 1.4 & 40 & 32 & $7.0 \times 10^{1}$ & $1.8 \times 10^{4}$ & $2.0 \times 10^{4}$ \\
\hline 18089-1732 & 3.7 & 6 & 180856.34 & -173219.6 & 12.6 & 30 & 31 & $1.7 \times 10^{3}$ & $2.8 \times 10^{5}$ & $5.2 \times 10^{4}$ \\
\hline $18110-1854$ & 4.1 & 2 & 181104.80 & -185423.1 & 7.8 & 30 & 34 & $1.1 \times 10^{3}$ & $1.4 \times 10^{5}$ & $9.6 \times 10^{4}$ \\
\hline 18116-1646 & 4.4 & 1 & 181141.85 & -164638.9 & 12.6 & 35 & 35 & $2.1 \times 10^{3}$ & $1.3 \times 10^{5}$ & $1.8 \times 10^{5}$ \\
\hline $18117-1753$ & 3.8 & 10 & 181144.37 & -175258.8 & 15.7 & 35 & 32 & $2.2 \times 10^{3}$ & $2.0 \times 10^{5}$ & $1.1 \times 10^{5}$ \\
\hline
\end{tabular}


Table 1. continued.

\begin{tabular}{|c|c|c|c|c|c|c|c|c|c|c|}
\hline \multirow[b]{3}{*}{ Source } & \multirow[b]{3}{*}{$\begin{array}{c}D \\
(\mathrm{kpc})\end{array}$} & \multicolumn{5}{|c|}{ Observed parameters } & \multicolumn{4}{|c|}{ Derived parameters } \\
\hline & & \multirow[b]{2}{*}{$N$} & \multicolumn{2}{|c|}{ Peak coordinates } & \multirow[b]{2}{*}{$\begin{array}{c}S_{1.2 \mathrm{~mm}} \\
(\mathrm{Jy})\end{array}$} & \multirow[b]{2}{*}{$\begin{array}{l}\theta_{\mathrm{S}} \\
\text { (") }\end{array}$} & \multirow[b]{2}{*}{$\begin{array}{l}T_{\mathrm{d}} \\
(\mathrm{K})\end{array}$} & \multirow[b]{2}{*}{$\begin{array}{c}M_{\mathrm{c}} \\
\left(M_{\odot}\right)\end{array}$} & \multirow[b]{2}{*}{$\begin{array}{c}n_{\mathrm{H}_{2}} \\
\left(\mathrm{~cm}^{-3}\right)\end{array}$} & \multirow[b]{2}{*}{$\begin{array}{c}L \\
\left(L_{\odot}\right)\end{array}$} \\
\hline & & & $\begin{array}{c}\alpha \\
(1950)\end{array}$ & $\begin{array}{c}\delta \\
(1950)\end{array}$ & & & & & & \\
\hline 18134-1942 & 1.7 & 1 & 181323.90 & -194231.7 & 9.0 & 48 & 29 & $2.9 \times 10^{2}$ & $1.1 \times 10^{5}$ & $6.8 \times 10^{3}$ \\
\hline $18139-1842$ & 4.1 & 1 & 181355.23 & -184244.4 & 2.6 & 32 & 32 & $4.2 \times 10^{2}$ & $4.2 \times 10^{4}$ & $5.8 \times 10^{4}$ \\
\hline 18159-1648 & 2.6 & 2 & 181600.92 & -164909.0 & 18.2 & 30 & 30 & $1.3 \times 10^{3}$ & $5.9 \times 10^{5}$ & $2.5 \times 10^{4}$ \\
\hline $18182-1433$ & 4.5 & 1 & 181818.37 & -143316.9 & 3.3 & 27 & 30 & $7.4 \times 10^{2}$ & $8.5 \times 10^{4}$ & $2.8 \times 10^{4}$ \\
\hline $18223-1243$ & 3.7 & 5 & 182222.08 & -124410.6 & 4.6 & 41 & 21 & $9.8 \times 10^{2}$ & $6.4 \times 10^{4}$ & $2.7 \times 10^{4}$ \\
\hline $18228-1312$ & 3.0 & 1 & 182253.00 & -131204.2 & 9.3 & 40 & 34 & $7.4 \times 10^{2}$ & $9.2 \times 10^{4}$ & $5.9 \times 10^{4}$ \\
\hline $18236-1205$ & 2.5 & 1 & 182337.41 & -120540.6 & 10.4 & 42 & 26 & $7.8 \times 10^{2}$ & $1.5 \times 10^{5}$ & $8.2 \times 10^{3}$ \\
\hline $18264-1152$ & 3.5 & 1 & 182626.69 & -115222.8 & 7.6 & 29 & 27 & $1.1 \times 10^{3}$ & $2.2 \times 10^{5}$ & $1.6 \times 10^{4}$ \\
\hline $18290-0924$ & 5.2 & 1 & 182857.77 & -09 2429.3 & 4.3 & 38 & 27 & $1.3 \times 10^{3}$ & $3.9 \times 10^{4}$ & $3.4 \times 10^{4}$ \\
\hline $18308-0503$ & 3.1 & 3 & 183049.81 & -050315.3 & 4.26 & 39 & 31 & $4.0 \times 10^{2}$ & $5.0 \times 10^{4}$ & $2.1 \times 10^{4}$ \\
\hline 18311-0809 & 9.2 & 3 & 183109.85 & -08 0944.6 & 8.0 & 39 & 31 & $6.6 \times 10^{3}$ & $3.2 \times 10^{4}$ & $3.6 \times 10^{5}$ \\
\hline $18314-0720$ & 5.9 & 1 & 183127.01 & -07 2018.9 & 16.2 & 69 & 32 & $5.3 \times 10^{3}$ & $1.7 \times 10^{4}$ & $4.4 \times 10^{5}$ \\
\hline $18316-0602$ & 3.1 & 1 & 183139.71 & -060154.3 & 10.6 & 34 & 30 & $1.0 \times 10^{3}$ & $1.9 \times 10^{5}$ & $2.9 \times 10^{4}$ \\
\hline $18317-0513$ & 3.2 & 1 & 183146.14 & $\begin{array}{lll}-05 & 13 & 12.9\end{array}$ & 7.7 & 37 & 31 & $7.7 \times 10^{2}$ & $1.0 \times 10^{5}$ & $2.7 \times 10^{4}$ \\
\hline $18317-0757$ & 4.9 & 1 & 183142.01 & -075708.5 & 8.1 & 39 & 35 & $1.7 \times 10^{3}$ & $5.1 \times 10^{4}$ & $1.4 \times 10^{5}$ \\
\hline $18341-0727$ & 6.4 & 3 & 183408.07 & -072723.2 & 13.4 & 46 & 29 & $5.9 \times 10^{3}$ & $5.1 \times 10^{4}$ & $1.9 \times 10^{5}$ \\
\hline $18411-0338$ & 6.3 & 1 & 184108.37 & -033825.8 & 5.2 & 33 & 33 & $1.9 \times 10^{3}$ & $4.7 \times 10^{4}$ & $1.6 \times 10^{5}$ \\
\hline $18434-0242$ & 7.4 & 6 & 184326.99 & -024232.6 & 11.1 & 26 & 39 & $4.6 \times 10^{3}$ & $1.4 \times 10^{5}$ & $1.1 \times 10^{6}$ \\
\hline 18440-0148 & 8.3 & 5 & 184400.58 & -014843.1 & 0.57 & 31 & 27 & $4.6 \times 10^{2}$ & $5.5 \times 10^{3}$ & $7.9 \times 10^{4}$ \\
\hline $18445-0222$ & 5.3 & 9 & 184433.43 & -02 2201.7 & 4.1 & 38 & 30 & $1.1 \times 10^{3}$ & $3.2 \times 10^{4}$ & $7.0 \times 10^{4}$ \\
\hline $18461-0113$ & 6.1 & 1 & 184606.94 & -011325.8 & 3.8 & 29 & 29 & $1.5 \times 10^{3}$ & $6.1 \times 10^{4}$ & $6.3 \times 10^{4}$ \\
\hline $18469-0132$ & 5.5 & 1 & 184656.92 & -013224.9 & 4.8 & 35 & 31 & $1.4 \times 10^{3}$ & $4.3 \times 10^{4}$ & $1.4 \times 10^{5}$ \\
\hline 18479-0005 & 13.0 & 1 & 184757.21 & -000531.7 & 11.6 & 27 & 38 & $1.5 \times 10^{4}$ & $7.7 \times 10^{4}$ & $1.4 \times 10^{6}$ \\
\hline $18502+0051$ & 7.1 & 1 & 185249.93 & 005521.5 & 14.7 & 36 & 30 & $7.5 \times 10^{3}$ & $1.0 \times 10^{5}$ & $2.0 \times 10^{5}$ \\
\hline $18507+0110$ & 3.7 & 1 & 185045.42 & 011114.3 & 58.8 & 59 & 37 & $6.3 \times 10^{3}$ & $1.5 \times 10^{5}$ & $7.5 \times 10^{5}$ \\
\hline $18507+0121$ & 3.7 & 3 & 185045.78 & 012115.0 & 13.2 & 47 & 28 & $2.0 \times 10^{3}$ & $9.2 \times 10^{4}$ & $3.4 \times 10^{4}$ \\
\hline $18517+0437$ & 3.0 & 1 & 185146.05 & 043750.1 & 6.7 & 33 & 32 & $5.5 \times 10^{2}$ & $1.3 \times 10^{5}$ & $2.2 \times 10^{4}$ \\
\hline $18530+0215$ & 5.1 & 1 & 185302.64 & 021516.3 & 4.8 & 35 & 31 & $1.2 \times 10^{3}$ & $4.7 \times 10^{4}$ & $9.0 \times 10^{4}$ \\
\hline $19078+0901$ & 11.8 & 3 & 190749.87 & 090119.9 & 105.7 & 33 & 30 & $1.5 \times 10^{5}$ & $4.0 \times 10^{4}$ & $5.6 \times 10^{6}$ \\
\hline $19095+0930$ & 3.3 & 1 & 190930.95 & 093047.6 & 5.3 & 28 & 34 & $5.0 \times 10^{2}$ & $1.4 \times 10^{5}$ & $4.2 \times 10^{4}$ \\
\hline $19097+0847$ & 4.3 & 1 & 190944.70 & 084659.3 & 8.2 & 37 & 30 & $1.5 \times 10^{3}$ & $8.9 \times 10^{4}$ & $4.4 \times 10^{4}$ \\
\hline
\end{tabular}


S. Faúndez et al.: SIMBA southern survey. I., Online Material p 6

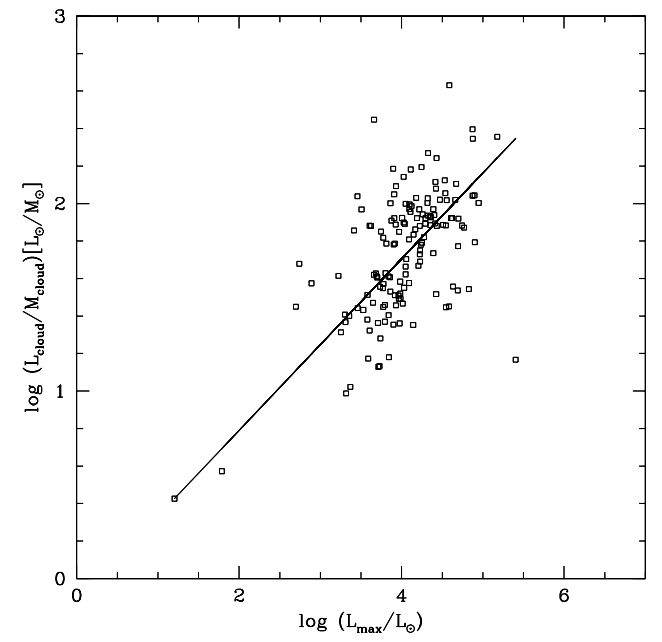

Fig. 6. Luminosity-to-mass ratio of the cores versus the luminosity of the most luminous star within the core. The equation of the straight line, fitted using a robust estimator, is $L_{\mathrm{c}} / M_{\mathrm{c}}=0.75\left(L_{\max }\right)^{0.46}$. The mean absolute deviation is 0.2 . 
S. Faúndez et al.: SIMBA southern survey. I., Online Material $p 7$
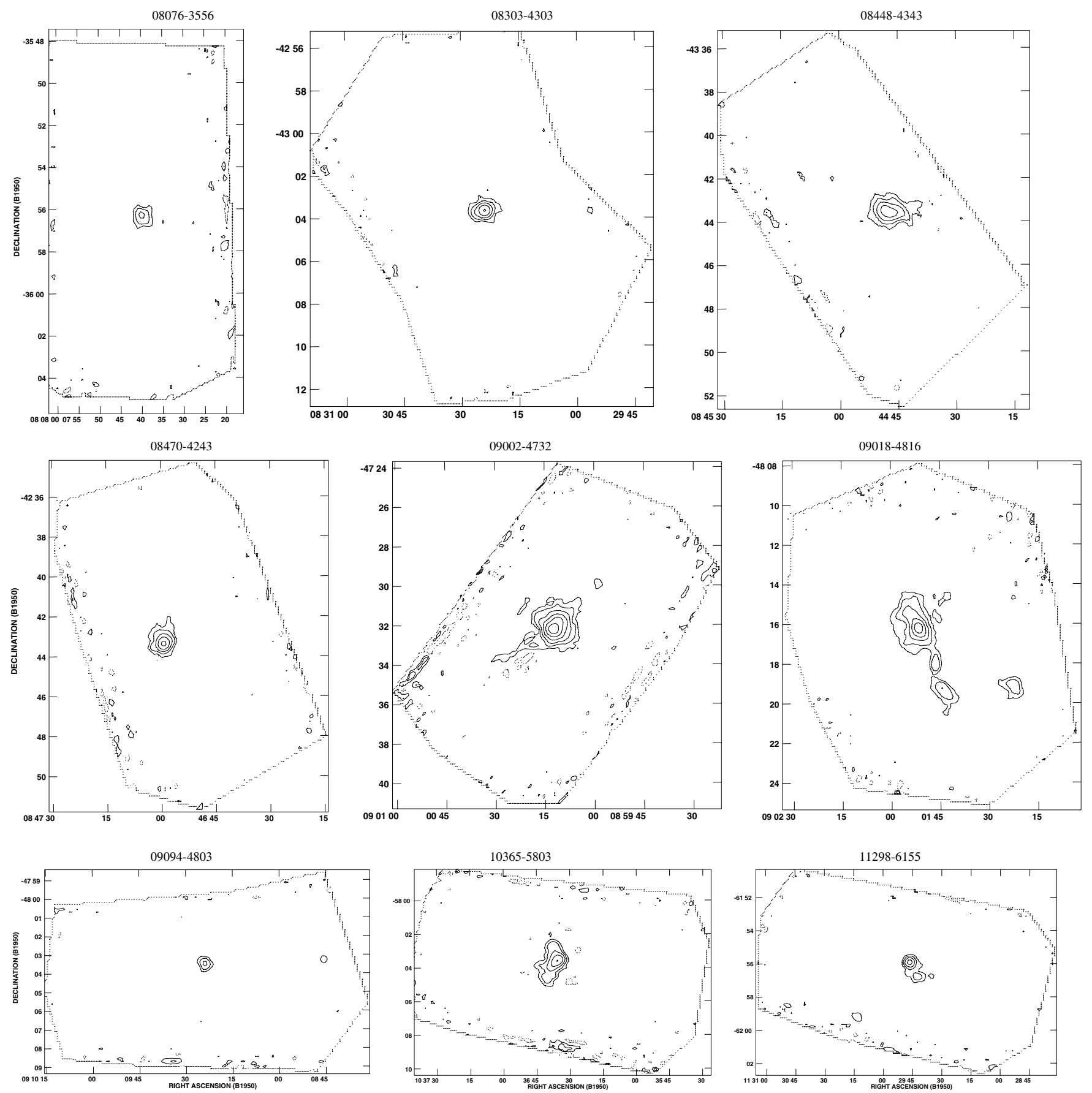

Fig. 7. Maps of the $1.2 \mathrm{~mm}$ emission IRAS name is given on top of each figure. Contour levels are $-3 \sigma, 3 \sigma, 6 \sigma, 12 \sigma, 24 \sigma, 48 \sigma \ldots$ Unless stated otherwise, $\sigma=40 \mathrm{mJy}$ for all sources. 
S. Faúndez et al.: SIMBA southern survey. I., Online Material p 8
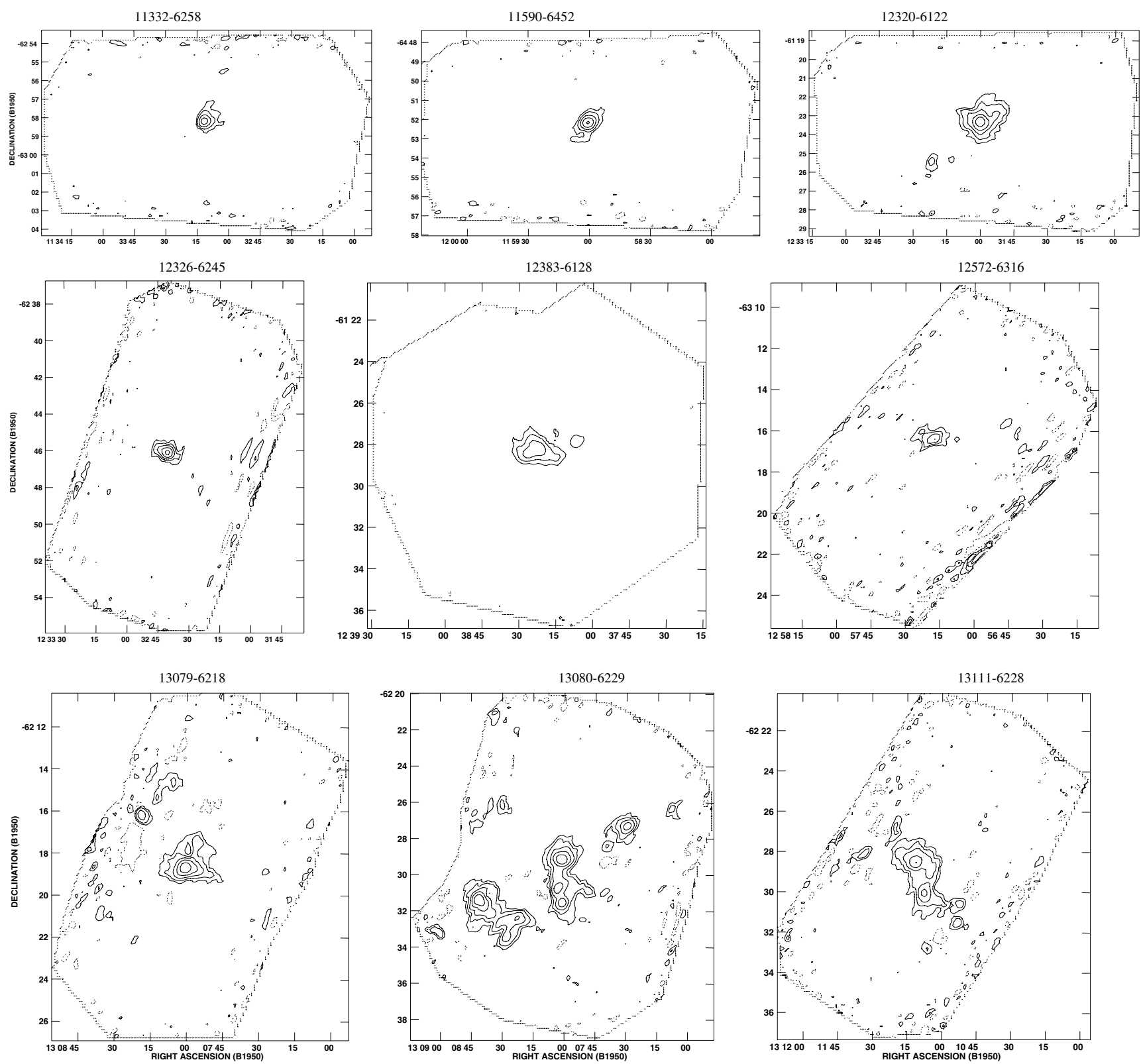

Fig. 8. Same as fig. 6. IRAS 12326-6245: $\sigma=200$ mJy, IRAS 12572-6316: $\sigma=50 \mathrm{mJy}$ and IRAS 13079-6218: $\sigma=60 \mathrm{mJy}$. 
S. Faúndez et al.: SIMBA southern survey. I., Online Material $p 9$
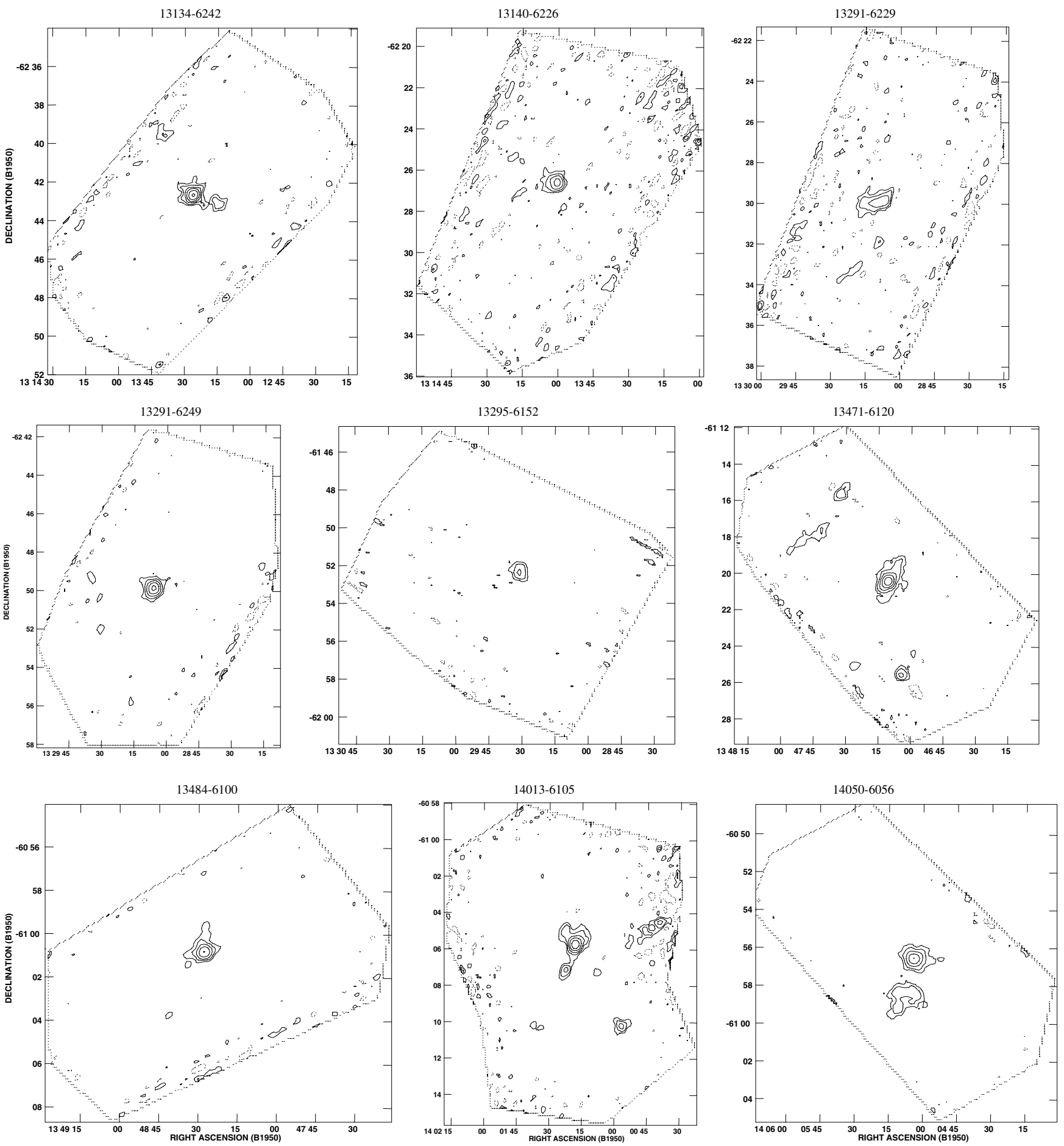

Fig. 9. Same as Fig. 6. IRAS 13295-6152: $\sigma=60 \mathrm{mJy}$. 
S. Faúndez et al.: SIMBA southern survey. I., Online Material p 10
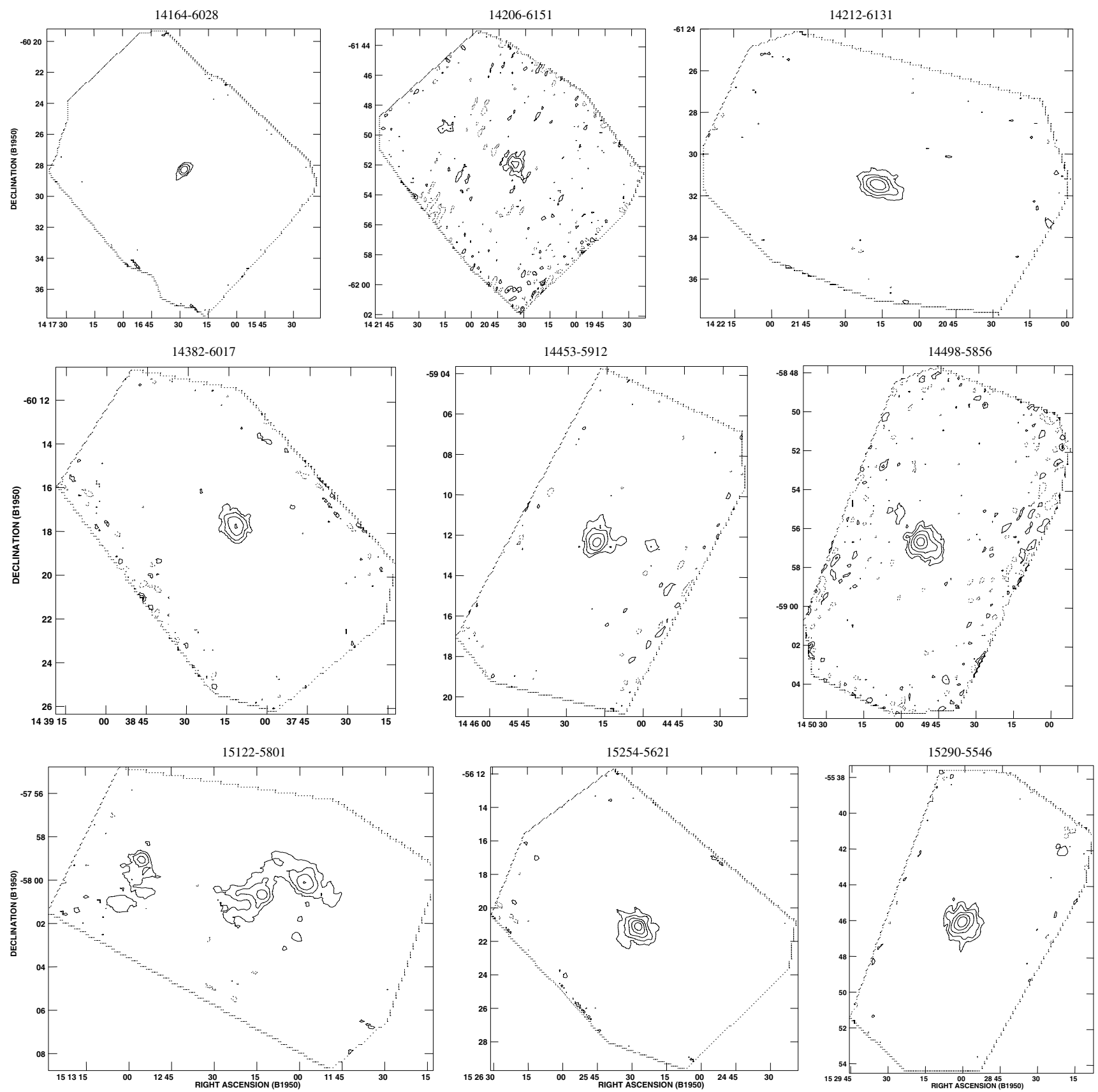

Fig. 10. Same as Fig. 6. 
S. Faúndez et al.: SIMBA southern survey. I., Online Material p 11
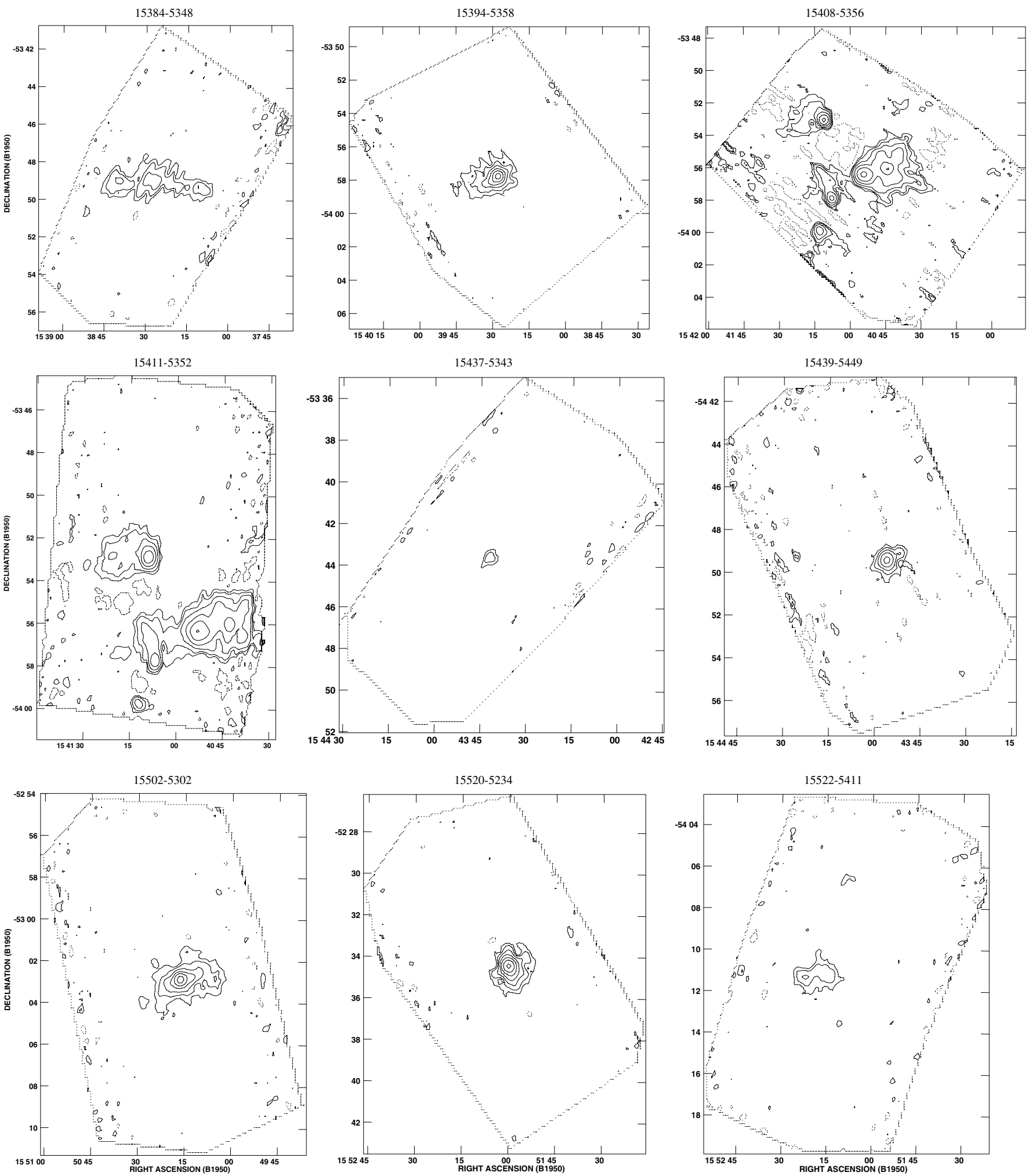

Fig. 11. Same as Fig. 6. IRAS 15384-5348: $\sigma=90 \mathrm{mJy}$, IRAS 15437-5343: $\sigma=130 \mathrm{mJy}$. 
S. Faúndez et al.: SIMBA southern survey. I., Online Material p 12
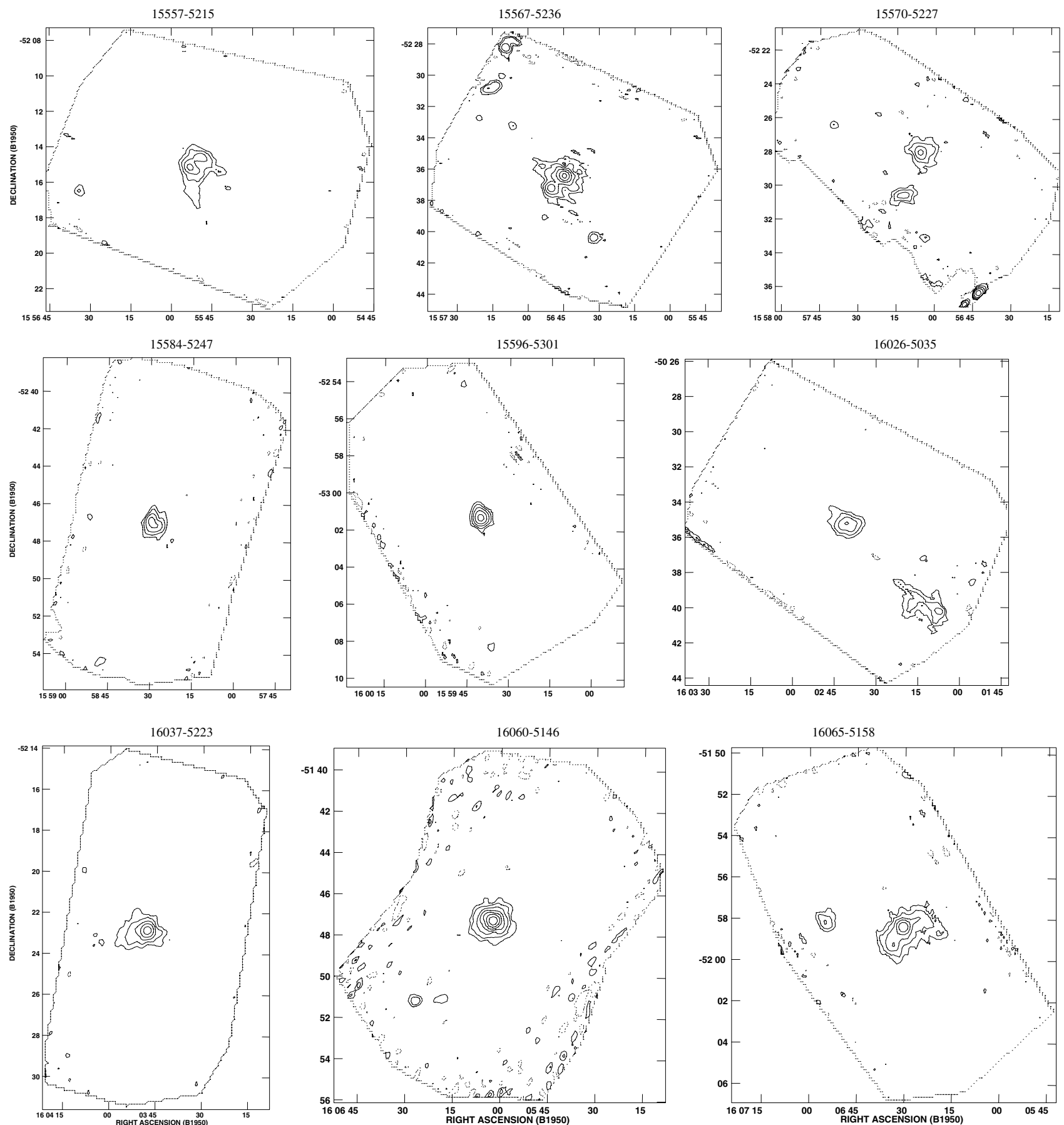

Fig. 12. Same as Fig. 6. IRAS 16060-5146 and 16065-5158: $\sigma=70 \mathrm{mJy}$. 
S. Faúndez et al.: SIMBA southern survey. I., Online Material p 13
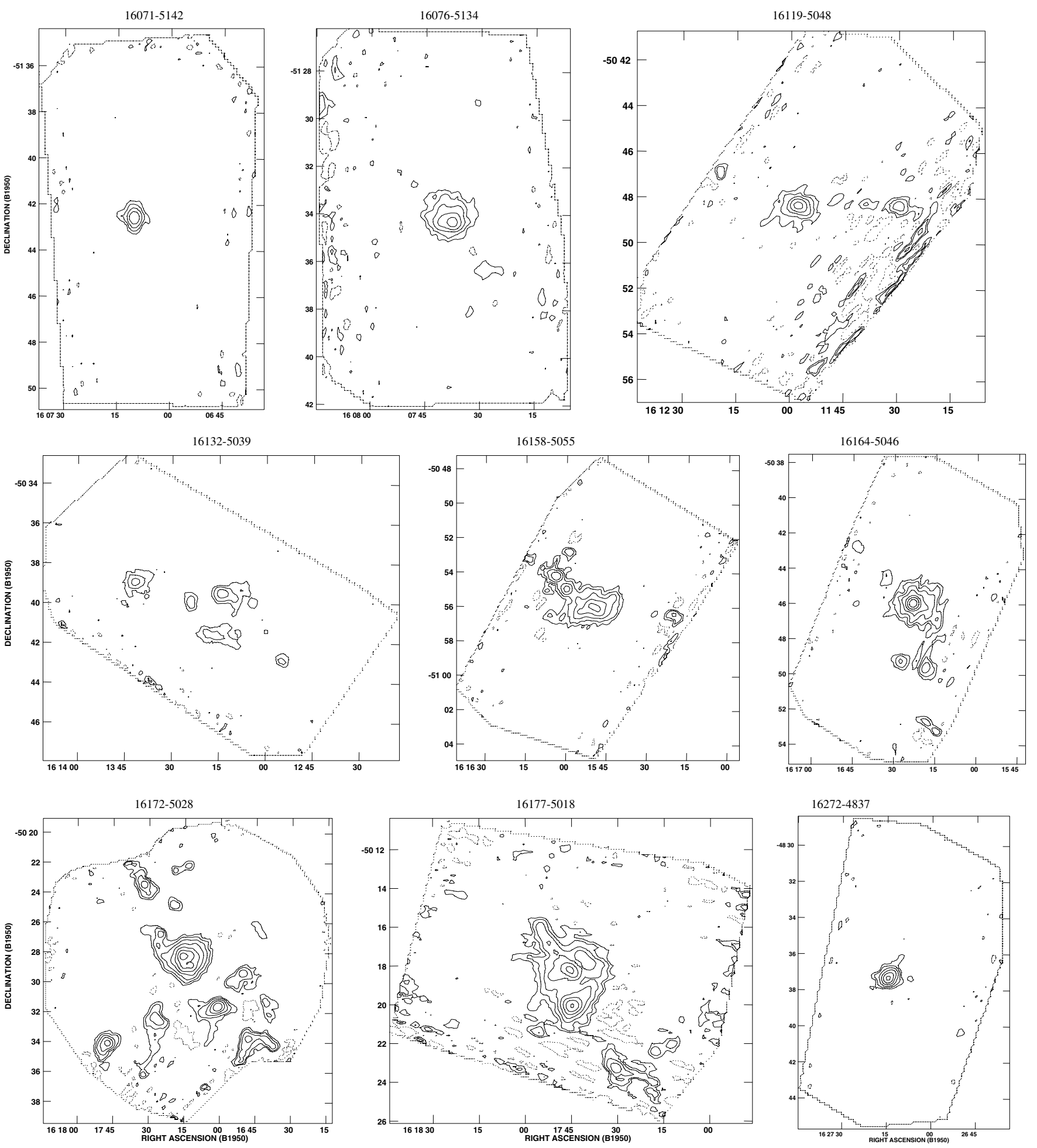

Fig. 13. Same as Fig. 6. IRAS 16071-5142: $\sigma=140 \mathrm{mJy}$. 
S. Faúndez et al.: SIMBA southern survey. I., Online Material $p 14$
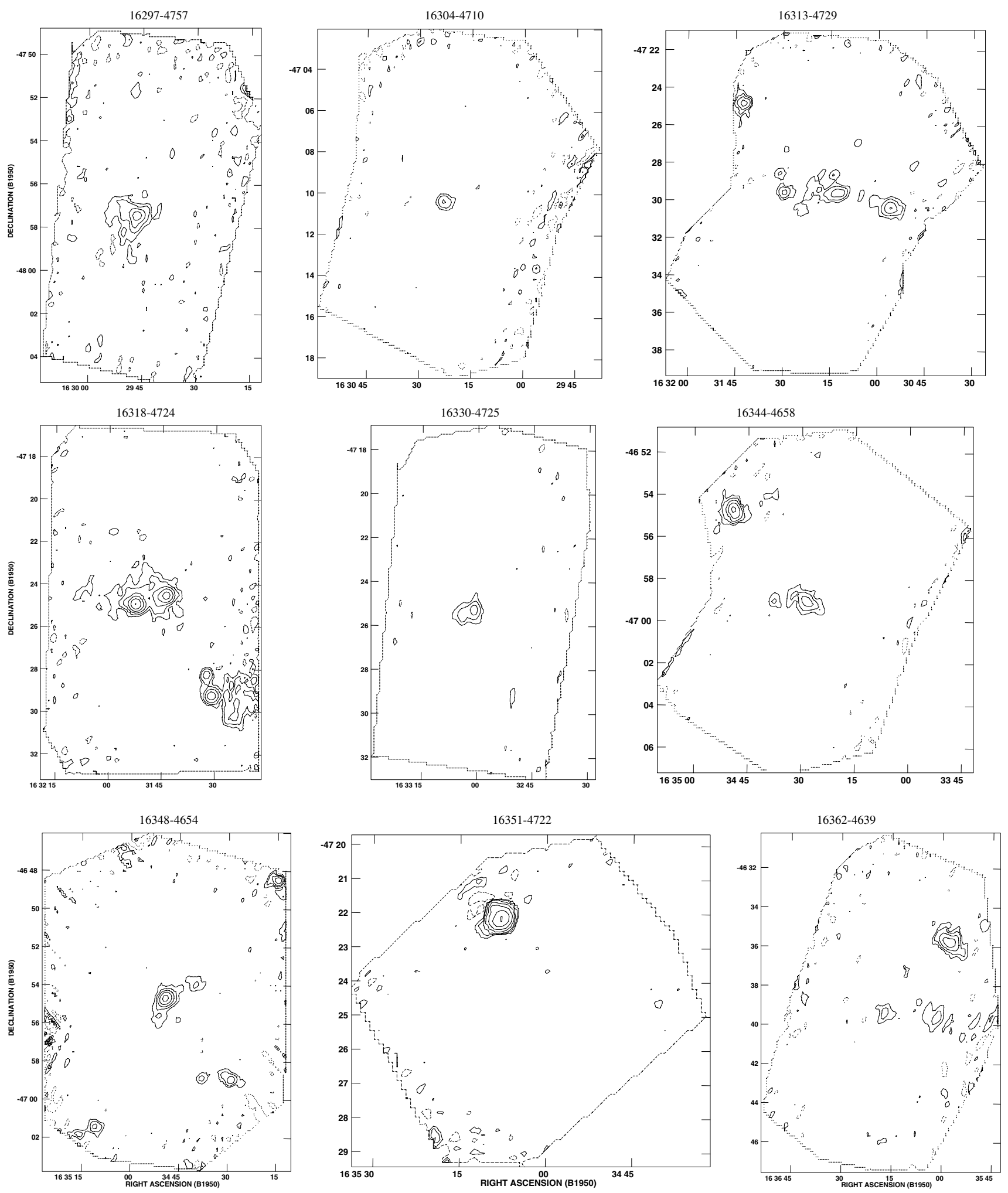

Fig. 14. Same as Fig. 6. IRAS 16304-4710: $\sigma=60$ mJy, IRAS 16313-4729 and 16348-4654: $\sigma=90$ mJy, IRAS 16330-4725: $\sigma=170$ mJy, IRAS 16344-4658: $\sigma=110 \mathrm{mJy}$ and IRAS 16362-4639: $\sigma=70 \mathrm{mJy}$. 
S. Faúndez et al.: SIMBA southern survey. I., Online Material $p 15$
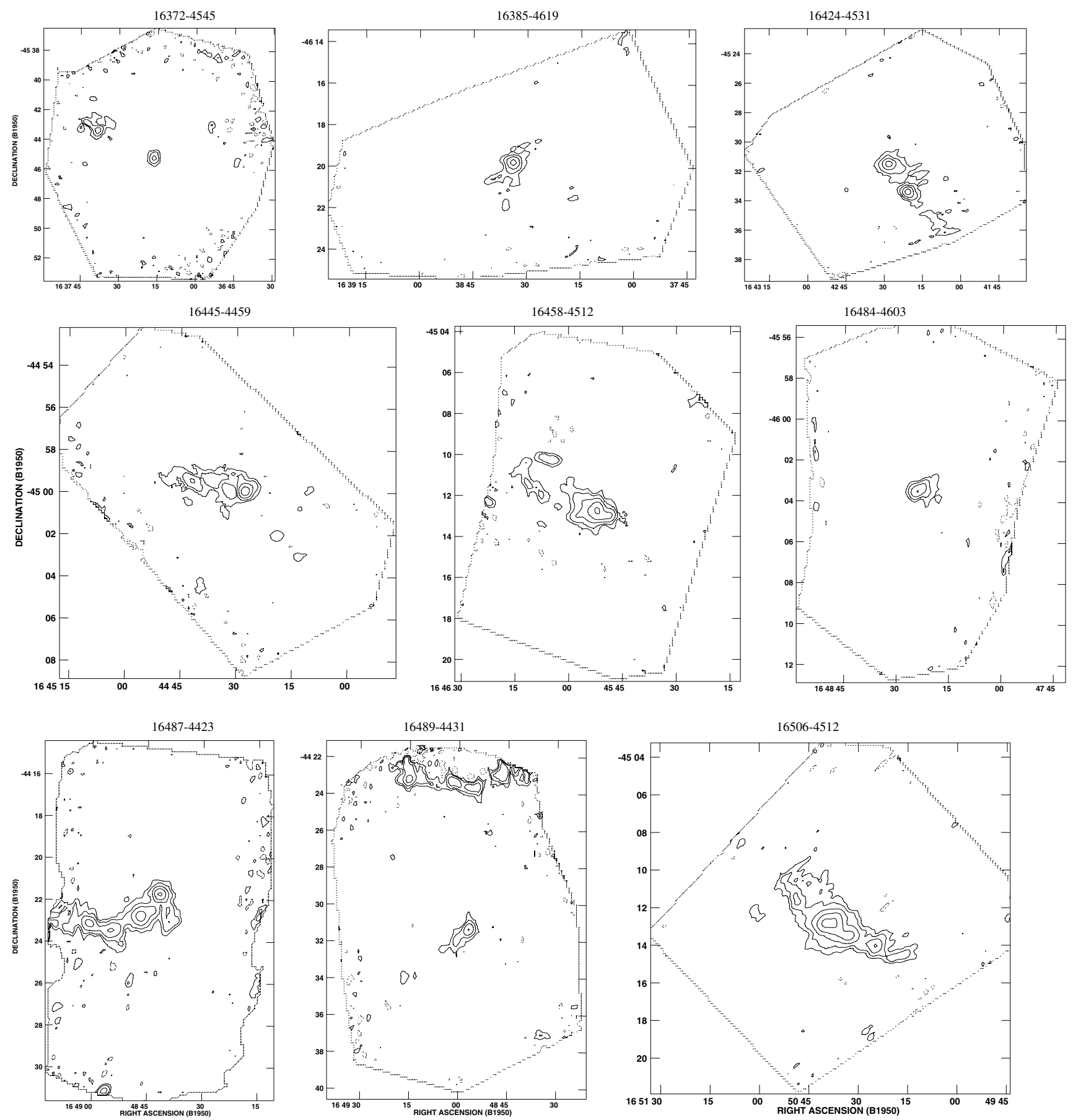

Fig. 15. Same as Fig. 6. IRAS 16372-4545: $\sigma=60 \mathrm{mJy}$ and IRAS 16484-4603: $\sigma=120 \mathrm{mJy}$. 
S. Faúndez et al.: SIMBA southern survey. I., Online Material p 16
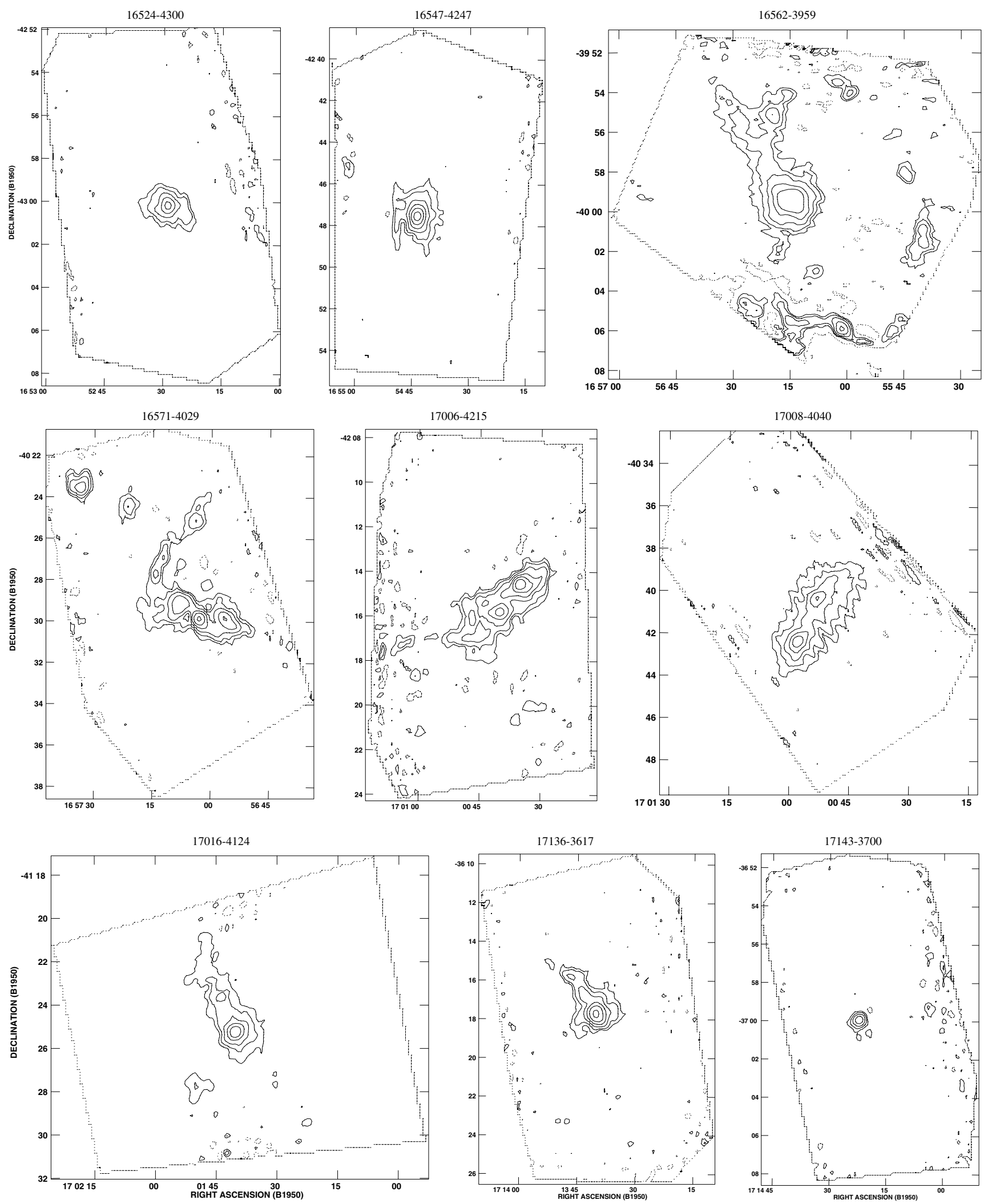

Fig. 16. Same than Fig. 6. IRAS $16562-3959$ has $\sigma=60$ mJy, IRAS 17008-4040 has $\sigma=110$ mJy and IRAS $17016-4124$ has $\sigma=80 \mathrm{mJy}$. 
S. Faúndez et al.: SIMBA southern survey. I., Online Material p 17
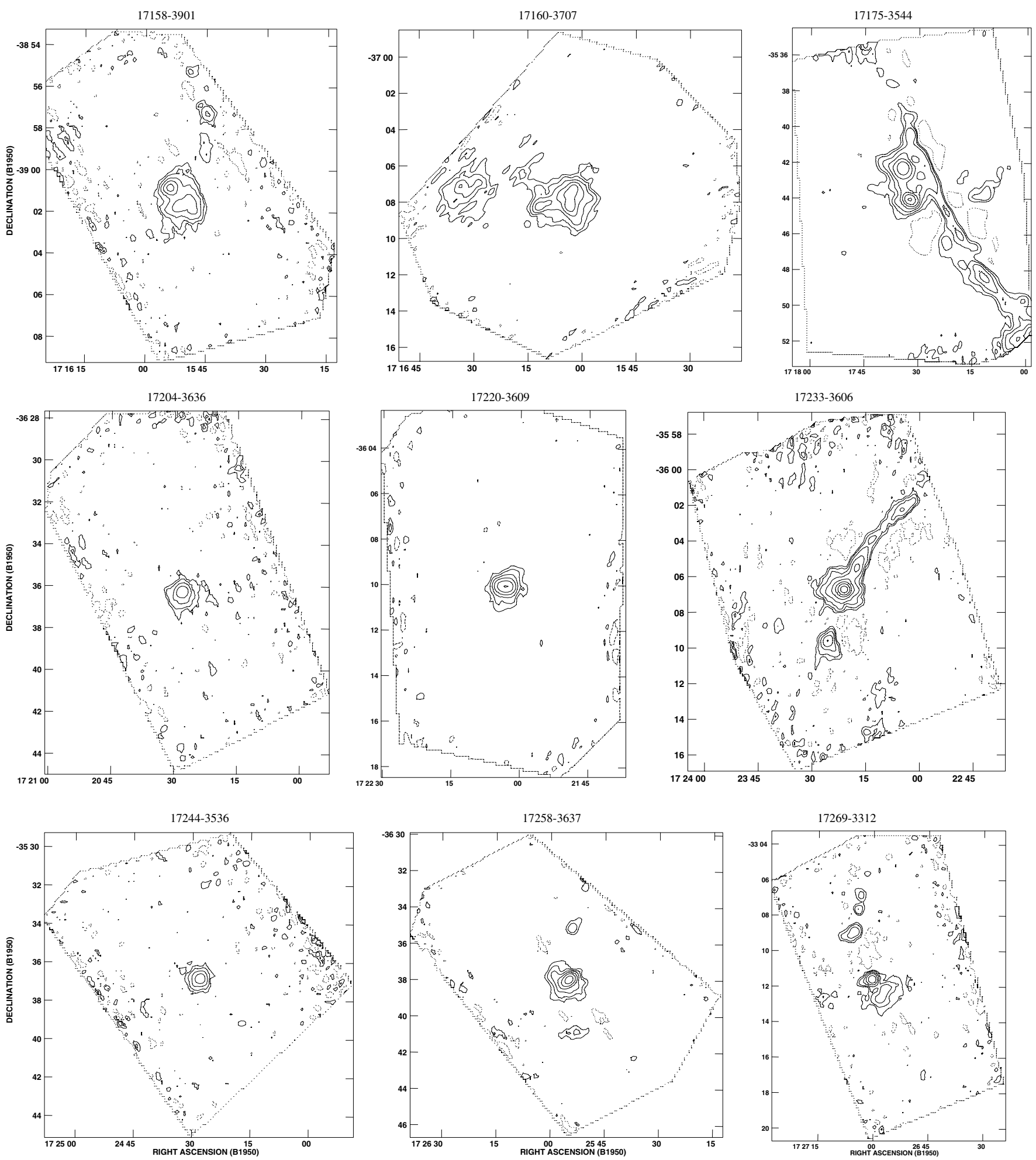

Fig. 17. Same as Fig. 6 IRAS 17175-3544, 17220-3609 and 17258-3637: $\sigma=90 \mathrm{mJy}$. 
S. Faúndez et al.: SIMBA southern survey. I., Online Material p 18
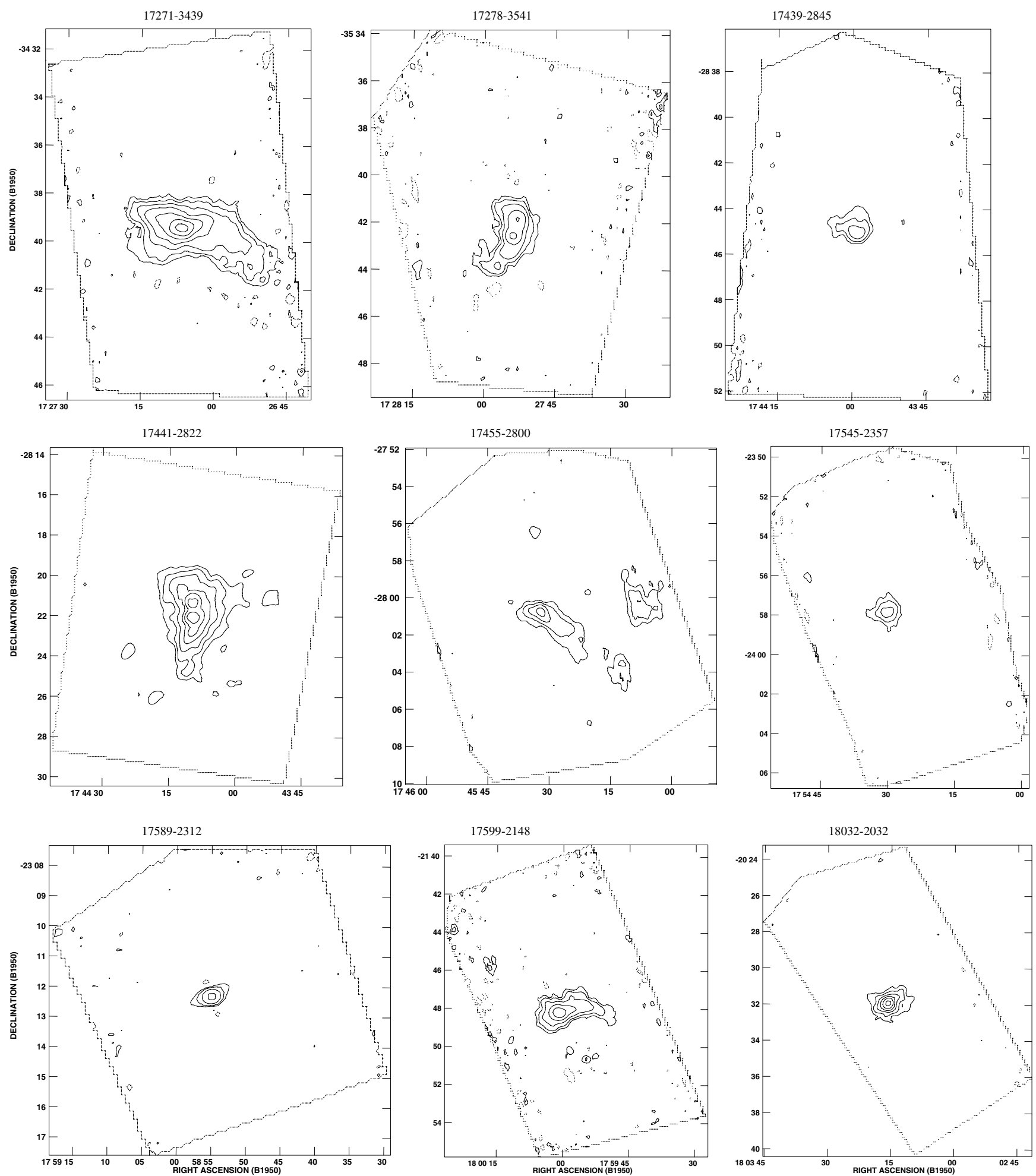

Fig. 18. Same as Fig. 6. IRAS 17439-2845, 17455-2800 and 17545-2357: $\sigma=90$ mJy. IRAS 17441-2822: $\sigma=300 \mathrm{mJy}$. 
S. Faúndez et al.: SIMBA southern survey. I., Online Material p 19
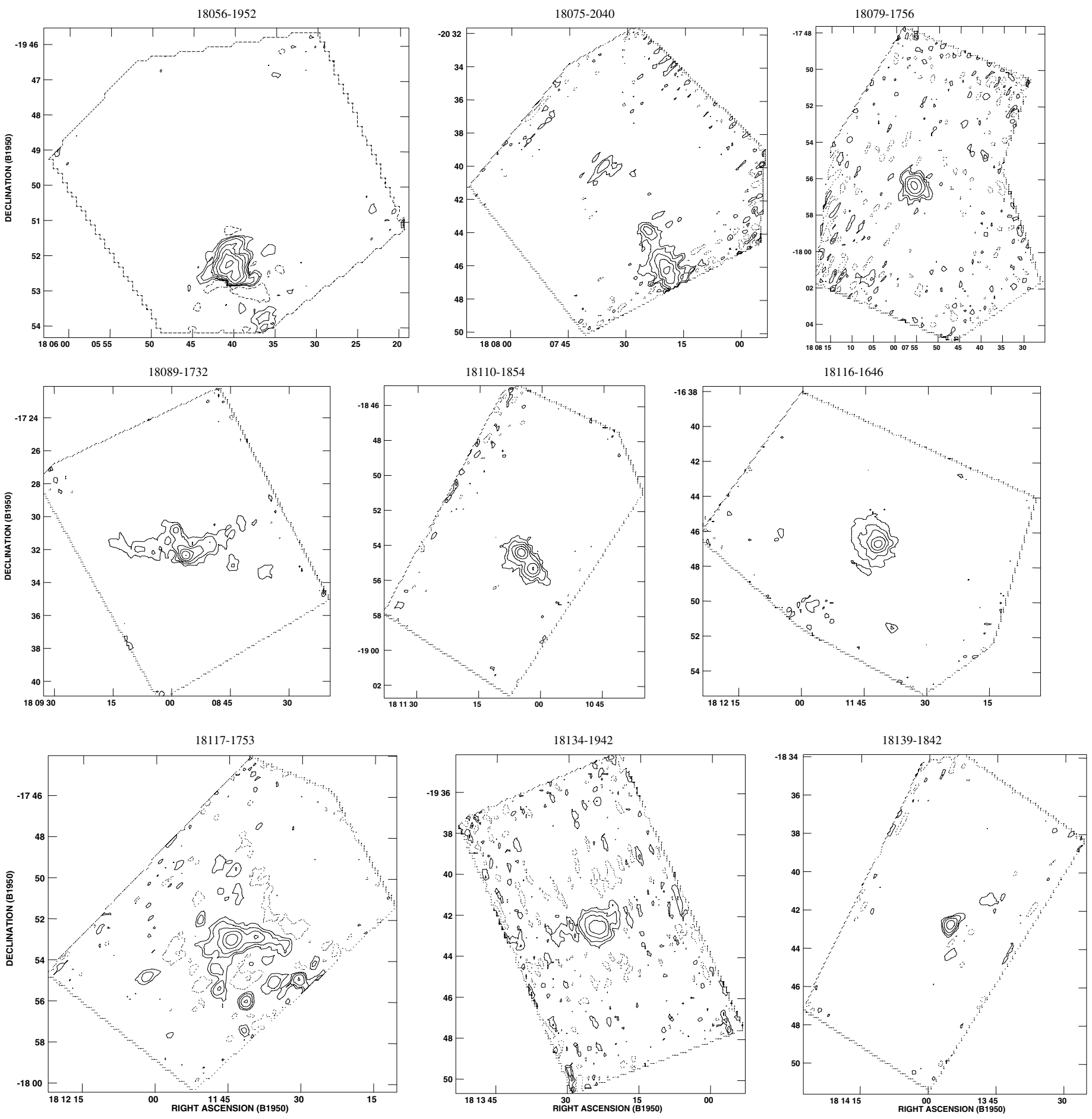

Fig. 19. Same as Fig. 6. IRAS 18075-2040: $\sigma=25 \mathrm{mJy}$. 
S. Faúndez et al.: SIMBA southern survey. I., Online Material p 20
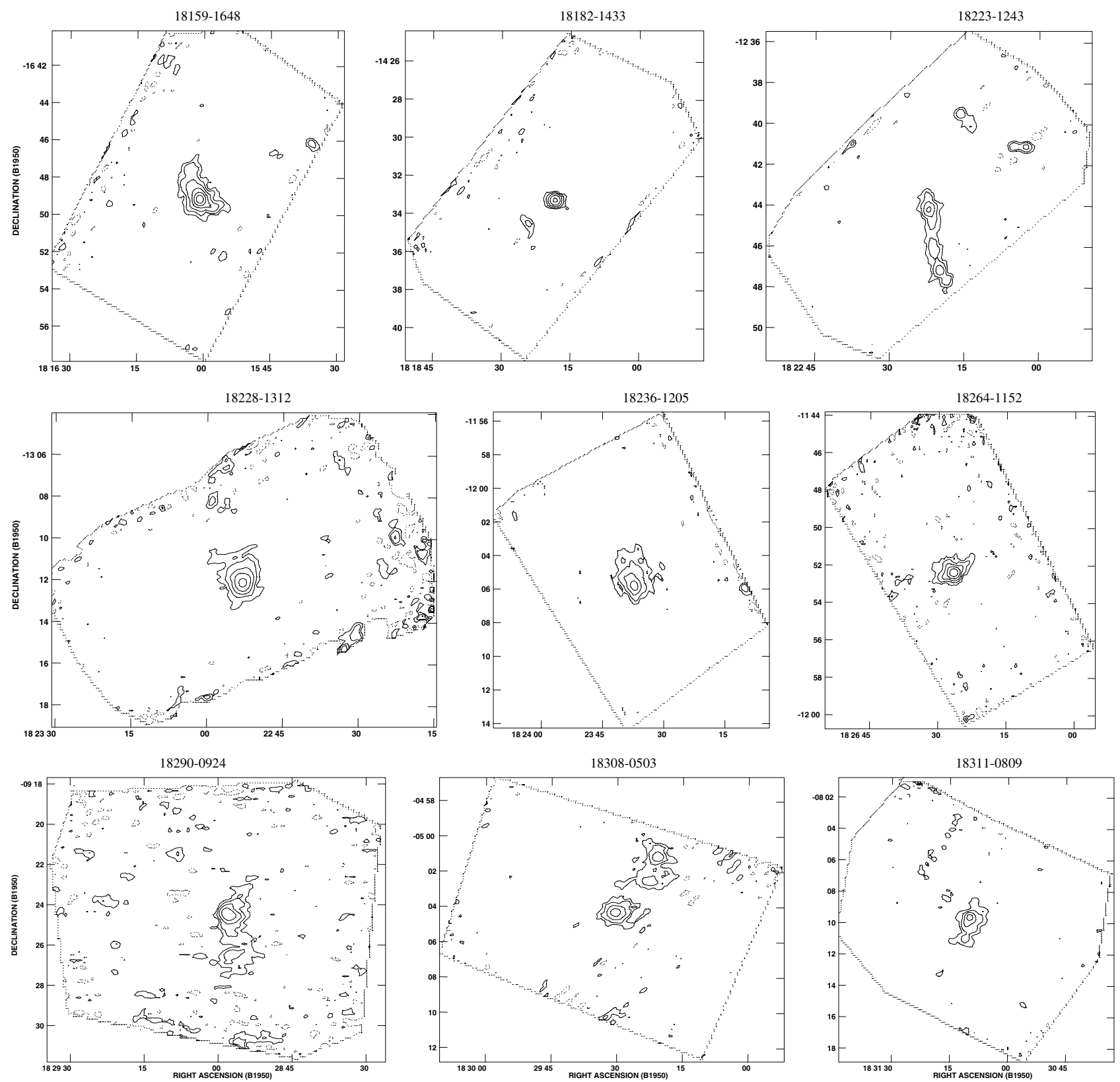

Fig. 20. Same as Fig. 6. IRAS 18290-0924: $\sigma=20 \mathrm{mJy}$ and IRAS 18308-0503: $\sigma=25 \mathrm{mJy}$. 
S. Faúndez et al.: SIMBA southern survey. I., Online Material p 21
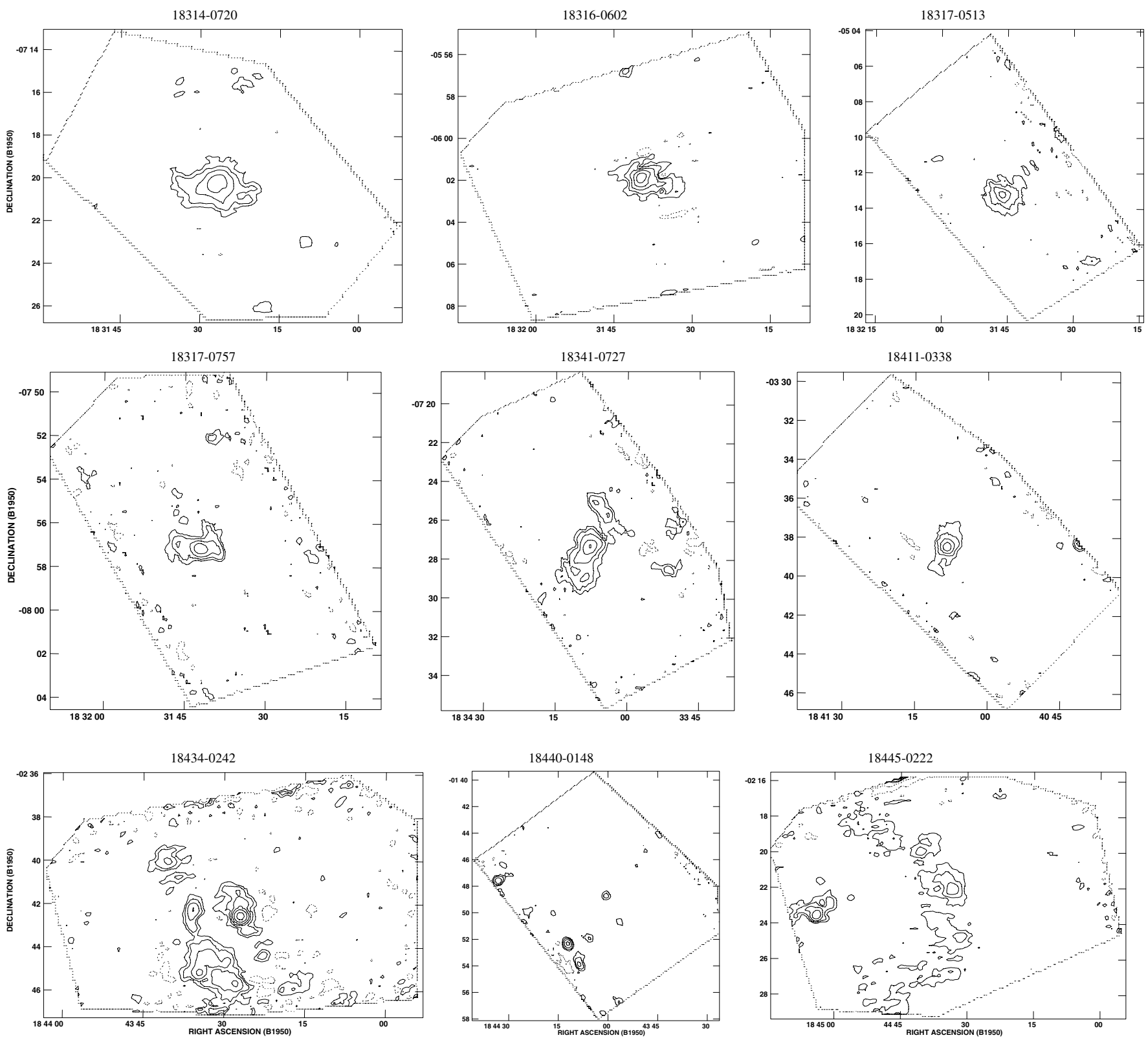

Fig. 21. Same as Fig. 6. 
S. Faúndez et al.: SIMBA southern survey. I., Online Material p 22
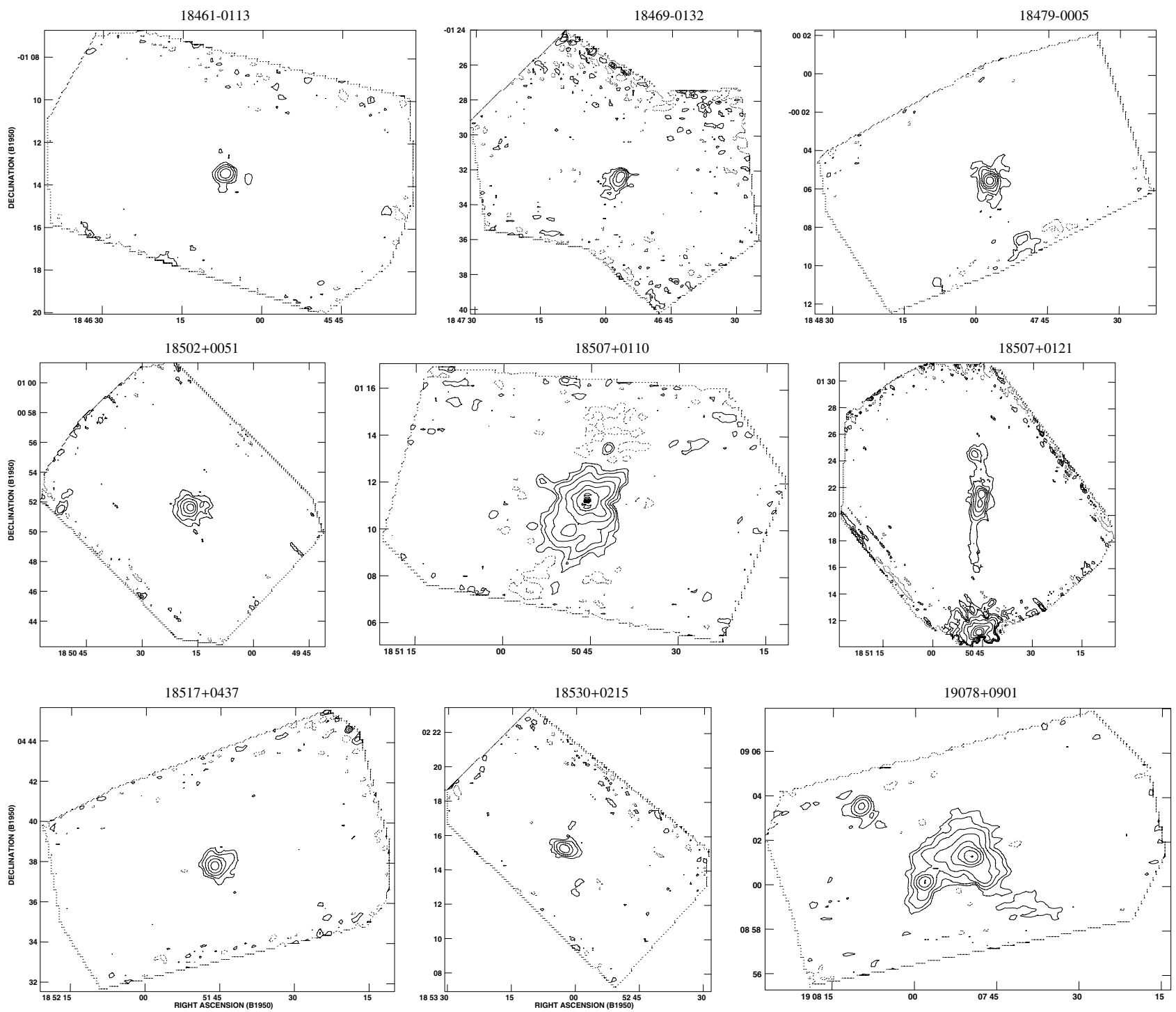

Fig. 22. Same as Fig. 6. IRAS 19078+0901: $\sigma=70 \mathrm{mJy}$.
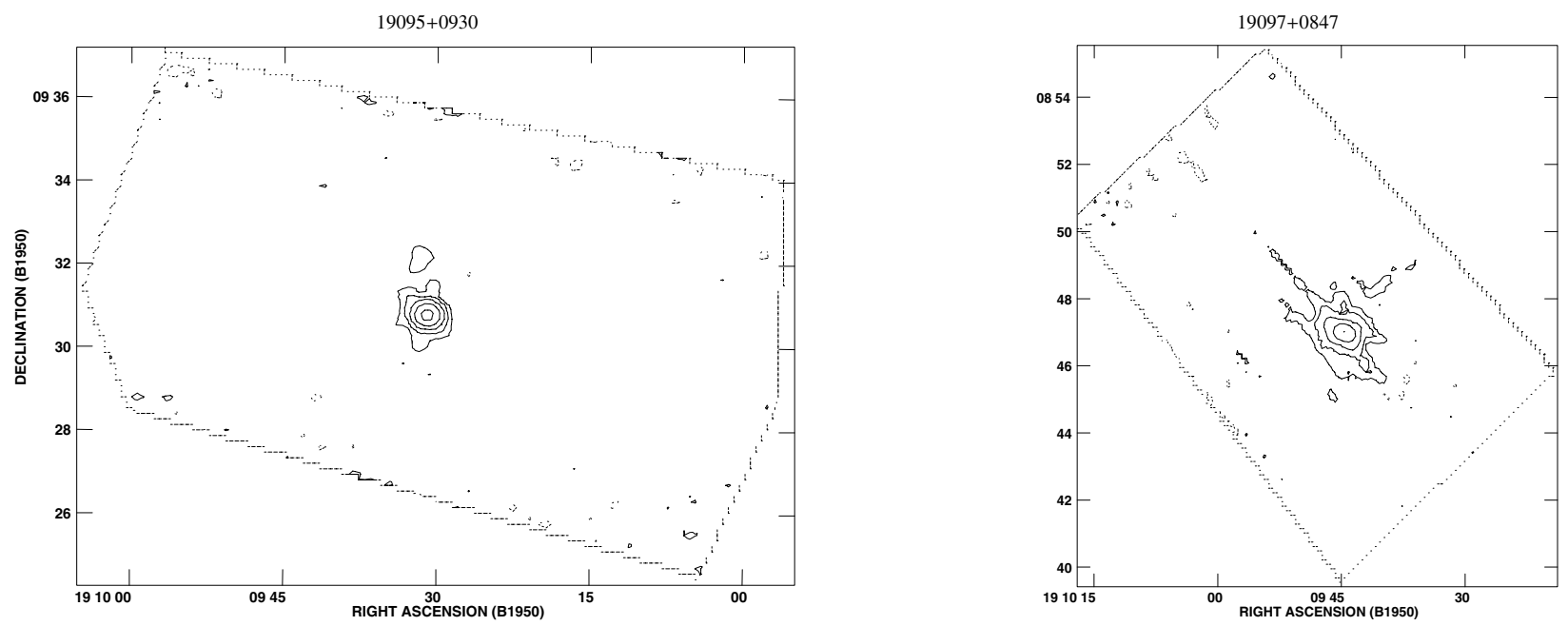

Fig. 23. Same as Fig. 6. 